\title{
Multiwavelength study of the star-formation in the bar of NGC 2903
}

\author{
G. Popping ${ }^{1}$, I. Pérez ${ }^{2,3}$, and A. Zurita ${ }^{2,3}$ \\ 1 Kapteyn Astronomical Institute, PO Box 800, 9700 AV Groningen, The Netherlands \\ e-mail: G. Popping@astro.rug.nl \\ 2 Dep. Física Teórica y del Cosmos, Campus de Fuentenueva, Universidad de Granada, 18071 Granada, Spain \\ 3 Instituto Carlos I de Física Teórica y Computación, Spain
}

Received 2 February 2010 / Accepted 28 April 2010

\begin{abstract}
Aims. The nearby barred spiral NGC 2903 has an active starburst at its centre and H II regions distributed along its bar. We analyse the star-formation properties in the bar region of NGC 2903 and study its links to the typical bar morphological features.

Methods. We combine space and ground-based data from the far-ultraviolet to the sub-millimeter spectral ranges to create a panchromatic view of the NGC 2903 bar. We produce two catalogues: one for the current star-formation regions, as traced by the H $\alpha$ compact emission, and a second for the ultraviolet (UV) emitting knots, containing positions and luminosities. From them, we obtain ultraviolet colours, star-formation rates, dust attenuation, and $\mathrm{H} \alpha E W \mathrm{~s}$, and analyse their spatial distribution. We estimate stellar cluster ages using stellar population synthesis models (Starburst 99 ).

Results. We find NGC 2903 to be a complex galaxy that has a very different morphology in each spectral band. The CO $(J=1-0)$ and the $3.6 \mu \mathrm{m}$ emission trace each other in a clear barred structure, while the $\mathrm{H} \alpha$ leads both components and has an s-shape distribution. The UV emission is patchy and does not resemble a bar. The UV emission is also characterised by a number of regions located symmetrically with respect to the galaxy centre, almost perpendicular to the bar, in a spiral shape covering the inner $\sim 2.5 \mathrm{kpc}$. These regions exhibit a significant amount of neither $\mathrm{H} \alpha$ nor $24 \mu \mathrm{m}$ emission. We estimate ages for these regions ranging from 150 to $320 \mathrm{Myr}$, older than the remaining UV knots, which have ages lower than $10 \mathrm{Myr}$. The SFR calculated from the UV emission is $\sim 0.4 M_{\odot} \mathrm{yr}^{-1}$, compatible with the SFR derived from $\mathrm{H} \alpha$ calibrations $\left(\sim 1 M_{\odot} \mathrm{yr}^{-1}\right)$.
\end{abstract}

Key words. galaxies: star formation - galaxies: structure - galaxies: individual: NGC 2903 - galaxies: evolution - HII regions ultraviolet: galaxies

\section{Introduction}

Following the launch of both the Spitzer Space Telescope and the GALaxy Evolution eXplorer (GALEX ), knowledge about the star-formation (SF) in galaxies has grown considerably. The panchromatic view of nearby galaxies offered by large surveys carried out by these space telescope missions have provided extraordinary databases with which to study star-formation in galaxies. In particular, it has allowed us to link general galaxy properties to those of the local interstellar medium (ISM) properties and the galaxy dynamics. Identifying these links is crucial for understanding SF in galaxies. In nearby galaxies, these sets of multiwavelength data provide an opportunity to study in detail, with good spatial resolution, the location and properties of relatively young populations, recent massive star-formation, and dust attenuation (e.g.; Calzetti et al. 2005; Tamura et al. 2009; Relaño \& Kennicutt 2009).

New dust and SF indicators have been developed and calibrated using all the available bands. The combination of $24 \mu \mathrm{m}$ and $\mathrm{H} \alpha$ emission has become a reliable indicator of dust attenuation (Kennicutt et al. 2007; Relaño \& Kennicutt 2009). Both bands are linked to star-formation processes, with the $\mathrm{H} \alpha$ emission originating from the recombination of hydrogen in the surrounding medium of very recently formed (less than a few Myr) massive stars, and the $24 \mu \mathrm{m}$ emission tracing ionising radiation from young stars obscured by dust (Calzetti et al. 2005). Extinction-corrected UV emission can also be used to retrieve the star-formation rate (SFR) (Kennicutt 1998) and to obtain some insight into the star-formation that has occurred in the past Gyr (e.g.; Bianchi et al. 2005; Hibbard et al. 2005). A combination of $\mathrm{UV}, \mathrm{H} \alpha$, and $24 \mu \mathrm{m}$ emission is indeed ideal for reconstructing the recent star-formation history of a galaxy by studying the location and properties of individual clusters and those of the gas and dust emission around them. To date, and to our knowledge, only a few galaxies have been analysed in this way; M 51 (Calzetti et al. 2005), M 81 (Pérez-González et al. 2006), M 33 (Relaño \& Kennicutt 2009; Verley et al. 2009), and NGC 7331 (Thilker et al. 2007).

Barred galaxies offer a useful tool for investigating the physical conditions that support star-formation in galaxies. The motions in bars are characterised by non-circular motions that push the gas into intersecting orbits, where shocks and star-formation can be triggered. The position and strength of the shocks are determined by the bar potential, the global dynamics within the bar region being driven by the bar. We now have a relatively good understanding of the gas behaviour under a bar potential (e.g.; Pérez et al. 2004) and this knowledge can be used to understand the conditions triggering star-formation. Bars and their surroundings host extreme physical conditions and a variety of ISM environments. They are perfect places to study the link between the conditions favouring star-formation and the galaxy dynamics. Therefore, a panchromatic view revealing the history of star-formation in bars can provide a unique insight into the 
Table 1. Log of ground-based $\mathrm{H} \alpha$ observations.

\begin{tabular}{ccccc}
\hline \hline Band & Date & Filter & Exp. time & Seeing \\
\hline $\mathrm{H} \alpha$ & 29 Oct 2007 & WFCH6568 & $3 \times 1200 \mathrm{~s}$ & $1.4^{\prime \prime}$ \\
$\mathrm{R}$ & 30 Oct. 2007 & HARRIS R & $3 \times 250 \mathrm{~s}$ & $2.1^{\prime \prime}$ \\
\hline
\end{tabular}

links between star-formation and the galaxy dynamics, which may also help us to understand how bars form and evolve.

We present a detailed multiwavelength study of the starformation in the bar region of NGC 2903 performed by analysing the correlations between the location and ages of the young stellar clusters, and the morphology of the bar. This was achieved in turn by analysing the emission in $\mathrm{H} \alpha$, UV, and $24 \mu \mathrm{m}$ as well as the optical data from the Sloan Digital Sky Survey (SDSS), in addition to $8 \mu \mathrm{m}$ and $\mathrm{CO}(J=1-0)$ emission.

We have chosen NGC 2903 for this research for a number of reasons: it is close-by $(8.9 \mathrm{Mpc}$; Drozdovsky \& Karachentsev 2000) allowing us to achieve high spatial resolution $\left(\sim 43 \mathrm{pc} \operatorname{arcsec}{ }^{-1}\right)$, and is isolated from large companions, preventing major merger effects in the results. Irwin et al. (2009) studied the H I content of NGC 2903 demonstrating that it possesses a large $\mathrm{H}$ I envelope of around three times its optical size. They also found a small H I companion $64 \mathrm{kpc}$ away from the galaxy in projection, which adds to a previously known small stellar companion. No clear sign of an interaction has been found so far.

NGC 2903 is an SBd galaxy showing a symmetric strong bar considered typical of this class of galaxies (Laurikainen \& Salo 2002). Previous observations have shown large amounts of $\mathrm{H} \alpha$ emission along the bar and not only at the ends of the bar and nuclear region (Sheth et al. 2002). The CO $(J=1-0)$, Spitzer, and GALEX data available makes this galaxy an ideal target for multiwavelength studies to retrieve insight into the star-formation history of bars.

A previous study by Leon et al. (2008) of the NGC 2903 bar showed that $\mathrm{HCN}(1-0)$ is distributed along the bar and at its centre. They compared the star-formation rate ratio of the bar to the centre with the predictions of numerical simulations by Martin \& Friedli (1997). This made them propose that the bar in NGC 2903 has an age between 200 and 600 Myr.

The outline of the paper is as follows. In Sect. 2, we present the observational data and in Sect. 3 we analyse the general morphology of NGC 2903. We present our methodology to obtain the bar H II regions and UV emission knots catalogues in Sect. 4. The following section contains the main results regarding the $E W_{\mathrm{H} \alpha}$, star-formation rates, UV colours, and ages of the stellar clusters. In Sect. 6, we discuss our main results and finally present a summary and conclusions.

\section{Observations and data reduction}

\section{1. $H_{\alpha}$ imaging}

The H $\alpha$ data of NGC 2903 was obtained with the Wide Field Camera at the $2.5 \mathrm{~m}$ Isaac Newton Telescope at the Roque de los Muchachos Observatory in October 2007. A summary of the observations is presented in Table 1. The galaxy was observed through a $95 \AA$ width narrowband filter with a total exposure time of $3600 \mathrm{~s}$, and an $R$-band filter, used for the continuum subtraction, with an exposure time of $750 \mathrm{~s}$. We carried out the overscan subtraction, bias, and flatfield correction using

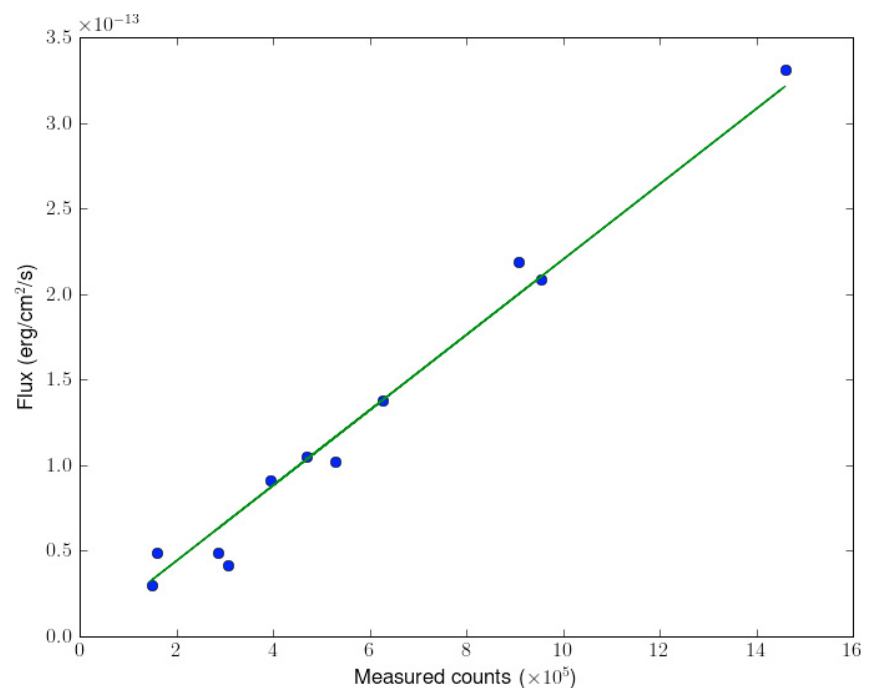

Fig. 1. Scaling relation from our measured $\mathrm{H} \alpha+[\mathrm{N}$ II] fluxes in counts to earlier measurements made by Mayya (1994) for the same H II regions. The H II region fluxes measured by Mayya (1994) also include those of the $[\mathrm{N}$ II] emission.

standard reduction tasks within IRAF ${ }^{1}$. The sky subtraction was carried out by fitting a first order polynomial to a sky map created using the mean sky values derived from several positions on the image, free of both foreground stars and galaxy emission. The $\mathrm{H} \alpha+$ continuum and $R$-band images were then aligned using the positions of field stars in the images and combined separately to produce the final images. Before continuum subtraction, the $\mathrm{H} \alpha+$ continuum image was degraded to the resolution of the $R$-band image $\left(\sim 2.1^{\prime \prime}\right)$.

The continuum subtraction was done as described by Relaño et al. (2005). We used 11 non-saturated foreground stars in both the $\mathrm{H} \alpha$ (ON-band) and in the continuum $R$-band (OFF-band) images. The fluxes of these stars yielded a mean flux ratio $\mathrm{ON}-$ band/OFF-band of $0.34 \pm 0.02$. This value was then used as a starting point scaling factor to generate a set of OFF-band images scaled with factors ranging from 0.32 to 0.36 . These images were afterwards subtracted from the $\mathrm{ON}$-band image to produce images free of continuum emission. After a close inspection of the resulting images, we adopted a value of $0.34 \pm 0.01$ for the continuum scaling factor. The uncertainty in the continuum scaling factor produces differences of up to $\sim 3 \%$ in the integrated fluxes of the $\mathrm{H}$ II regions.

The astrometry of the $\mathrm{H} \alpha$ image was performed using the USNO2 catalogue coordinates for the foreground stars of the galaxy images, resulting in an accuracy of $\sim 0.39^{\prime \prime}$. The observations were made during non-photometric conditions. Therefore, for the flux calibration, we used previously reported fluxes of 11 H II regions located in the disk of NGC 2903 published by Mayya (1994). We compared the flux reported there with our measured flux (in ADU) using identical apertures. We then calculated the ratio for each region and assumed the median to be our final $\mathrm{H} \alpha$ flux calibration factor $((2.2 \pm 0.4) \times$ $10^{-19} \mathrm{erg} \mathrm{s}^{-1} \mathrm{~cm}^{-2}$ count $\left.^{-1}\right)$. The error was calculated by taking the standard deviation of the flux ratio for all the stars. The scaling relation is presented in Fig. 1 . The $2 \sigma$ sensitivity limit of the final $\mathrm{H} \alpha$ image is $6.2 \times 10^{-17} \mathrm{erg} \mathrm{s}^{-1} \mathrm{~cm}^{-2}$ per pixel.

\footnotetext{
1 IRAF is distributed by the National Optical Astronomy Observatories, which is operated by the Association of Universities for Research in Astronomy, Inc. (AURA) under cooperative agreement with the National Science Foundation.
} 
The $\mathrm{H} \alpha$ data of Mayya (1994) from which our fluxes were flux calibrated were not corrected for Galactic extinction. Therefore, the HII region luminosities of our catalogued H II regions were corrected a posteriori for foreground Galactic extinction (Schlegel et al. 1998), which was assumed to be $A_{\mathrm{H} \alpha}=0.08 \mathrm{mag}$.

The bandwidth of the $\mathrm{H} \alpha$ filter can also be affected by emission from the [N II] $\lambda 6548 \AA$ and [N II] $\lambda 6584 \AA$ emission lines at their corresponding redshifted wavelengths. A proper correction for this requires spectroscopic information about all the H II regions, which information is not currently available. The typical [N II]/H $\alpha$ ratio for extragalactic $\mathrm{H}$ II regions of solar metallicity is approximately 0.33 (e.g.; Kennicutt \& Kent 1983). Therefore, an approximate correction of $25 \%$ should be applied to the $\mathrm{H} \alpha$ fluxes and equivalent widths. Rather than applying this average correction to the fluxes of all $\mathrm{H}$ II regions, we decided to present the uncorrected $\mathrm{H} \alpha+[\mathrm{N}$ II] luminosities in Table 2.

\subsection{Spitzer infrared images}

To investigate the extinction and the star-formation in the bar of NGC 2903 we used the Spitzer $24 \mu \mathrm{m}$ observations from the Multiband Imaging Photometer (MIPS) instrument (Werner et al. 2004; Fazio et al. 2004; Rieke et al. 2004). The data was obtained in April 2004 as part of the Local Volume Legacy Survey (Lee et al. 2008; Kennicutt et al. 2007). MIPS reduction steps are described in detail by Gordon et al. (2005). The field of view covered by the observations is large enough to contain the whole galaxy with enough sky coverage allowing a good sky subtraction. The $24 \mu \mathrm{m}$ point-spread function (PSF) has a measured $F W H M$ of $\sim 5.6^{\prime \prime}$ and a $2 \sigma$ sensitivity limit of $0.52 \times 10^{-6} \mathrm{Jy} \mathrm{arcsec}^{-2}$. The image was registered to the same coordinate system and pixel scale as the $\mathrm{H} \alpha$ image and the background was subtracted in the same way as for the $\mathrm{H} \alpha$ image.

The $24 \mu \mathrm{m}$ image has a much larger PSF $F W H M$ than the $\mathrm{H} \alpha$ image $\left(F W H M \sim 2.1^{\prime \prime}\right)$. Therefore, to allow for accurate comparisons, we produced a convolved version of the $\mathrm{H} \alpha$ image corresponding to the $24 \mu \mathrm{m}$ PSF by applying a PSF kernel as described by Gordon et al. (2008). This version image was only used to calculate the $\mathrm{H} \alpha$ attenuation of the $\mathrm{H}$ II regions (Sect. 4.3). As complementary data, we also used the 3.6 and $8 \mu \mathrm{m}$ images, which have a PSF FWHM $\sim 2^{\prime \prime}$ in both bands. These images were only used to carry out global morphological comparisons with the remaining bands; we therefore registered the images to the same coordinate system as the $\mathrm{H} \alpha$ image, but we did not degrade their spatial resolution.

\subsection{GALEX images}

To analyse the UV emission and its distribution in NGC 2903, we used GALEX mission data (Martin et al. 2005). The GALEX direct imaging observations were centered on $1529 \AA$ and $2312 \AA$ for the far-ultraviolet (FUV, 1350-1750 ) and nearultraviolet (NUV, 1750-2750 A) bands, respectively. NGC 2903 was observed in February 2004 as part of the Nearby Galaxies Survey (NGS, Bianchi et al. 2003) with an exposure time of $1862 \mathrm{~s}$ and $1861 \mathrm{~s}$ for the FUV and NUV, respectively. The PSF $F W H M$ of the images are $~ 4.2$ and $\sim 4.6$ " for the FUV and NUV, respectively, and their respective sensitivity limits $5.25 \times 10^{-18}$ and $3.09 \times 10^{-18} \mathrm{erg} \mathrm{s}^{-1} \mathrm{~cm}^{-2} \AA^{-1}$ per pixel (see Bianchi et al. 2003 for further details on the NGC 2903 GALEX data).

Both the FUV and NUV images were aligned to the same coordinate system and pixel scale as the $\mathrm{H} \alpha$ image and the background was subtracted in the same way as for the $\mathrm{H} \alpha$ and Spitzer data.

\subsection{Other data: ground-based optical images and $\mathrm{CO}(\mathrm{J}=1-0)$}

Ground-based optical images from the Sloan Digital Sky Survey (SDSS) and a map of the CO $(J=1-0)$ rotational transition emission were also used for the morphological analysis of NGC 2903. The optical data set comprises $g^{\prime}, r^{\prime}$, and $z^{\prime}$ band images, which were observed as part of the SDSS data release 6 and have a PSF $F W H M$ of $\sim 1.1^{\prime \prime}$. We subtracted the sky emission from these images using the same IRAF script used for the $\mathrm{H} \alpha$ image (Sect. 2.1).

For the photometric calibration, we estimated the calibration factor for converting the image digital counts to calibrated $g^{\prime}$, $r^{\prime}$, and $z^{\prime} \mathrm{AB}$ magnitudes as described on the SDSS web page for the data release $6^{2}$. These calibrated images were used to produce a $g^{\prime}-z^{\prime}$ colour map of NGC 2903 (Fig. 2e).

The CO $(J=1-0)$ emission-line map of NGC 2903 was used to trace the molecular gas emission. It was obtained from the BIMA (Berkeley-Illinois-Maryl and Association) array and the NRAO (National Radio Astronomy Observatory) ${ }^{3} 12 \mathrm{~m}$ single-dish telescope, as part of the BIMA SONG key project. The emission-line map has a maximum $F W H M$ of $6.8^{\prime \prime}$. Data acquisition and reduction details can be found in Regan et al. (2001) and Helfer et al. (2003).

\section{The general morphology of NGC 2903}

The different appearance of galaxies in different wavelength ranges has already been illustrated by many authors. Galaxies in the UV bands seem to be patchier and of later type than the same galaxies observed at optical and near-infrared wavelengths. Stellar components such as bulges, bars, and old stellar disks tend to disappear when observed in the UV range (e.g.; Kuchinski et al. 2000). NGC 2903 provides a good example of this behaviour. Figure 2 shows NGC 2903 observed in different wavelength regimes: FUV, NUV, $3.6 \mu \mathrm{m}, 24 \mu \mathrm{m}, g^{\prime}-z^{\prime}$ colour map and a $\mathrm{CO}(J=1-0)$ rotational transition emission-line map. All images contain superimposed $\mathrm{H} \alpha$ contours.

The UV distribution is patchy and spiral-like and does not resemble the smooth bar-like distribution shown in the $3.6 \mu \mathrm{m}$ image. The CO $(J=1-0)$ traces the $3.6 \mu \mathrm{m}$ emission, while the $\mathrm{H} \alpha$ follows an s-shape distribution that is also followed by the main dust-lanes, as shown by the $g^{\prime}-z^{\prime}$ colour image. Dust spurs emerge from the main dust lanes at many locations.

The $\mathrm{H} \alpha$ emission leads the CO $(J=1-0)$ emission, taking into account that NGC 2903 rotates counter-clockwise (Hernandez et al. 2005), assuming trailing spiral arms.

\section{Photometry of star-forming regions}

\subsection{HII region catalogue}

To study the properties of the recent star-forming sites in the NGC 2903 bar, we produced a catalogue of the H II regions located in the bar zone (except the nucleus). We consider the bar zone to be the area covering the whole lense region (Kormendy 1979) as observed in the $3.6 \mu \mathrm{m}$ image (see Fig. 2c).

\footnotetext{
2 http://www.sdss.org/dr6/algorithms/fluxcal . html

3 The National Radio Astronomy Observatory is a facility of the NSF operated under cooperative agreement by Associated Universities, Inc.
} 
Table 2. H II region catalogue of the bar zone of NGC 2903.

\begin{tabular}{|c|c|c|c|c|c|c|c|c|c|c|}
\hline ID & $\begin{array}{c}\text { RA } \\
(\mathrm{J} 2000)\end{array}$ & $\begin{array}{c}\text { Dec } \\
(\mathrm{J} 2000)\end{array}$ & $\begin{array}{l}\text { Radius } \\
(\operatorname{arcsec})\end{array}$ & $e$ & $\begin{array}{c}\text { PA } \\
(\mathrm{deg})\end{array}$ & $\begin{array}{c}\log L_{\mathrm{H}_{\alpha}+[\mathrm{NIII}]} \\
\left(\mathrm{in} \mathrm{erg} \mathrm{s}^{-1} \text { ) }\right.\end{array}$ & $\begin{array}{c}\log E W_{\mathrm{H}_{\alpha}+[\mathrm{NII}]} \\
(\mathrm{in} \AA)\end{array}$ & $\begin{array}{c}\log \left(L_{24 \mu \mathrm{m}}\right) \\
\left(\mathrm{erg} \mathrm{s}^{-1}\right)\end{array}$ & $\begin{array}{c}A_{\mathrm{H} \alpha} \\
(\mathrm{mag})\end{array}$ & $\begin{array}{c}S F R \\
\left(10^{-3} M_{\odot} \mathrm{yr}^{-1}\right)\end{array}$ \\
\hline 1 & $09: 32: 12.35$ & $21: 31: 04.66$ & 3.1 & 0 & 90 & $38.99 \pm 0.05$ & $2.32 \pm 0.11$ & 40.60 & 1.21 & $17.7 \pm 0.9$ \\
\hline 2 & 09:32:11.69 & $21: 30: 40.84$ & 5.23 & 0.51 & 158 & $39.41 \pm 0.05$ & $2.86 \pm 0.02$ & 40.93 & 1.07 & $41.0 \pm 2.2$ \\
\hline 3 & 09:32:11.67 & $21: 30: 12.75$ & 3.17 & 0 & 0 & $38.71 \pm 0.10$ & - & 40.24 & 1.09 & $8.3 \pm 0.9$ \\
\hline 4 & $09: 32: 12.56$ & $21: 30: 29.23$ & 3 & 0 & 0 & $38.75 \pm 0.08$ & $2.70 \pm 0.09$ & 40.19 & 0.95 & $7.9 \pm 0.8$ \\
\hline 5 & 09:32:08.77 & $21: 29: 39.86$ & 7.3 & 0.81 & -28 & $39.28 \pm 0.08$ & $2.34 \pm 0.04$ & 40.71 & 0.93 & $26.5 \pm 2.9$ \\
\hline 6 & 09:32:07.62 & $21: 29: 33.54$ & 3.1 & 0 & 0 & $38.81 \pm 0.08$ & $3.07 \pm 0.03$ & 40.35 & 1.09 & $10.5 \pm 0.9$ \\
\hline 7 & 09:32:14.38 & $21: 30: 40.38$ & 4.23 & 0.68 & 6 & $38.76 \pm 0.12$ & - & 40.24 & 1.01 & $8.6 \pm 1.2$ \\
\hline 8 & 09:32:13.75 & 21:31:02.56 & 5.23 & 0.8 & -48 & $38.87 \pm 0.11$ & - & 39.49 & 0.21 & $5.3 \pm 1.5$ \\
\hline 9 & $09: 32: 13.83$ & 21:31:09.62 & 2.33 & 0 & 0 & $38.43 \pm 0.10$ & $2.50 \pm 0.01$ & 39.32 & 0.36 & $2.2 \pm 0.5$ \\
\hline 10 & 09:32:12.57 & $21: 30: 52.67$ & 4.17 & 0.77 & 10 & $38.60 \pm 0.14$ & - & 40.05 & 0.96 & $5.7 \pm 1.0$ \\
\hline 11 & 09:32:13.60 & $21: 30: 24.86$ & 3.7 & 0.52 & 38 & $38.62 \pm 0.14$ & - & 40.01 & 0.88 & $5.6 \pm 1.1$ \\
\hline 12 & 09:32:07.12 & $21: 29: 26.37$ & 2.03 & 0 & 0 & $38.12 \pm 0.16$ & $2.28 \pm 0.01$ & 39.65 & 1.08 & $2.1 \pm 0.4$ \\
\hline 13 & 09:32:11.43 & 21:30:00.36 & 3.1 & 0 & 0 & $38.55 \pm 0.14$ & - & 39.97 & 0.92 & $4.9 \pm 0.9$ \\
\hline 14 & 09:32:12.44 & $21: 30: 40.75$ & 3 & 0 & 0 & $38.70 \pm 0.09$ & $2.43 \pm 0.14$ & 40.13 & 0.93 & $7.0 \pm 0.8$ \\
\hline 15 & 09:32:12.63 & $21: 30: 36.70$ & 2.23 & 0 & 0 & $38.30 \pm 0.13$ & - & 39.70 & 0.90 & $2.7 \pm 0.5$ \\
\hline 16 & 09:32:05.59 & 21:28:54.01 & 2.8 & 0 & 90 & $38.59 \pm 0.10$ & $2.87 \pm 0.03$ & 39.77 & 0.61 & $4.1 \pm 0.7$ \\
\hline 17 & 09:32:07.77 & $21: 28: 56.02$ & 3.93 & 0 & 0 & $38.87 \pm 0.11$ & - & 40.51 & 1.27 & $14.1 \pm 1.4$ \\
\hline 18 & 09:32:07.56 & 21:29:11.66 & 2.6 & 0 & 0 & $38.33 \pm 0.16$ & - & 40.23 & 1.74 & $6.4 \pm 0.6$ \\
\hline 19 & 09:32:07.83 & 21:29:26.69 & 3.57 & 0 & 90 & $38.66 \pm 0.14$ & - & 40.32 & 1.30 & $9.0 \pm 1.2$ \\
\hline 20 & 09:32:12.01 & $21: 30: 17.87$ & 2.87 & 0.85 & 3 & $38.22 \pm 0.13$ & - & 39.79 & 1.14 & $2.8 \pm 0.4$ \\
\hline 21 & 09:32:12.35 & $21: 30: 22.63$ & 2.7 & 0.78 & 108 & $38.25 \pm 0.13$ & $2.60 \pm 0.09$ & 39.49 & 0.68 & $2.0 \pm 0.4$ \\
\hline 22 & 09:32:13.76 & $21: 30: 18.72$ & 1.9 & 0 & 0 & $38.00 \pm 0.18$ & - & 39.22 & 0.66 & $1.1 \pm 0.3$ \\
\hline 23 & 09:32:11.44 & 21:31:11.46 & 2.33 & 0 & 90 & $38.30 \pm 0.14$ & - & 39.87 & 1.15 & $3.4 \pm 0.5$ \\
\hline 24 & 09:32:08.76 & $21: 28: 46.76$ & 3.53 & 0 & 90 & $39.10 \pm 0.05$ & $2.31 \pm 0.04$ & 40.50 & 0.89 & $16.8 \pm 1.2$ \\
\hline 25 & 09:32:07.88 & 21:28:47.69 & 3.93 & 0 & 0 & $39.02 \pm 0.07$ & $2.63 \pm 0.01$ & 40.59 & 1.15 & $18.0 \pm 1.4$ \\
\hline 26 & 09:32:11.67 & $21: 31: 27.14$ & 3.6 & 0 & 0 & $38.77 \pm 0.11$ & - & 40.39 & 1.23 & $10.8 \pm 1.2$ \\
\hline 27 & 09:32:09.59 & $21: 28: 47.12$ & 4.17 & 0 & 0 & $39.41 \pm 0.03$ & $2.44 \pm 0.02$ & 40.93 & 1.08 & $40.8 \pm 1.6$ \\
\hline 28 & 09:32:10.62 & 21:31:31.74 & 3.5 & 0 & 90 & $38.62 \pm 0.15$ & $2.56 \pm 0.04$ & 40.20 & 1.17 & $7.2 \pm 1.1$ \\
\hline 29 & 09:32:10.18 & $21: 28: 42.26$ & 3.1 & 0 & 90 & $38.52 \pm 0.15$ & $2.31 \pm 0.03$ & 40.13 & 1.21 & $6.0 \pm 0.9$ \\
\hline 30 & 09:32:08.53 & $21: 31: 30.16$ & 2.53 & 0 & 0 & $38.46 \pm 0.11$ & $2.44 \pm 0.06$ & 40.10 & 1.27 & $5.5 \pm 0.6$ \\
\hline 31 & 09:32:11.20 & $21: 28: 57.28$ & 3.67 & 0 & 0 & $39.19 \pm 0.04$ & $2.67 \pm 0.03$ & 40.59 & 0.89 & $20.7 \pm 1.2$ \\
\hline 32 & 09:32:11.67 & $21: 28: 30.75$ & 3.7 & 0 & 0 & $38.84 \pm 0.10$ & $2.67 \pm 0.07$ & 39.62 & 0.29 & $5.3 \pm 1.3$ \\
\hline 33 & $09: 32: 12.57$ & $21: 28: 38.22$ & 3.5 & 0 & 0 & $39.14 \pm 0.04$ & $2.76 \pm 0.02$ & 40.56 & 0.91 & $19.1 \pm 1.1$ \\
\hline 34 & 09:32:13.09 & $21: 28: 43.61$ & 3.83 & 0 & 0 & $39.24 \pm 0.04$ & $2.49 \pm 0.03$ & 40.19 & 0.41 & $14.8 \pm 1.4$ \\
\hline 35 & 09:32:07.93 & $21: 28: 16.18$ & 2.67 & 0 & 0 & $38.34 \pm 0.17$ & $2.63 \pm 0.00$ & 39.73 & 0.88 & $2.9 \pm 0.7$ \\
\hline 36 & 09:32:09.63 & 21:31:06.38 & 6.4 & 0 & 90 & $39.81 \pm 0.03$ & $2.51 \pm 0.02$ & 41.47 & 1.29 & $126.1 \pm 3.8$ \\
\hline 37 & 09:32:08.43 & 21:31:42.49 & 2.8 & 0 & 90 & $38.61 \pm 0.10$ & - & 39.67 & 0.50 & $3.8 \pm 0.7$ \\
\hline 38 & 09:32:08.81 & 21:30:55.01 & 4 & 0 & 0 & $39.14 \pm 0.06$ & $2.52 \pm 0.08$ & 40.51 & 0.85 & $17.8 \pm 1.5$ \\
\hline 39 & 09:32:09.08 & 21:31:14.75 & 2.17 & 0 & 0 & $38.35 \pm 0.11$ & - & 40.07 & 1.41 & $4.9 \pm 0.4$ \\
\hline 40 & $09: 32: 12.38$ & $21: 31: 30.57$ & 2.33 & 0 & 0 & $38.30 \pm 0.14$ & - & 38.98 & 0.24 & $1.5 \pm 0.5$ \\
\hline 41 & 09:32:11.97 & 21:31:21.00 & 2 & 0 & 0 & $38.22 \pm 0.12$ & - & 39.68 & 0.98 & $2.4 \pm 0.4$ \\
\hline 42 & 09:32:08.06 & $21: 28: 42.43$ & 3.67 & 0 & 0 & $38.87 \pm 0.09$ & $2.25 \pm 0.01$ & 40.32 & 0.97 & $10.7 \pm 1.2$ \\
\hline 43 & 09:32:13.40 & $21: 28: 46.21$ & 3.83 & 0 & 0 & $39.16 \pm 0.05$ & $2.41 \pm 0.03$ & 40.20 & 0.48 & $13.4 \pm 1.4$ \\
\hline 44 & 09:32:09.01 & 21:28:40.08 & 2.37 & 0 & 0 & $38.52 \pm 0.09$ & - & 40.38 & 1.67 & $9.2 \pm 0.5$ \\
\hline 45 & 09:32:11.96 & 21:29:10.88 & 3.27 & 0 & 0 & $38.90 \pm 0.07$ & $2.64 \pm 0.02$ & 40.55 & 1.27 & $15.3 \pm 1.0$ \\
\hline 46 & 09:32:10.39 & 21:31:11.67 & 3.7 & 0 & 0 & $38.79 \pm 0.11$ & $2.43 \pm 0.04$ & 40.42 & 1.25 & $11.5 \pm 1.3$ \\
\hline 47 & 09:32:13.05 & 21:31:35.10 & 2.43 & 0 & 0 & $38.28 \pm 0.16$ & $2.85 \pm 0.01$ & 39.45 & 0.62 & $2.0 \pm 0.5$ \\
\hline 48 & 09:32:13.47 & 21:31:32.35 & 2.7 & 0 & 0 & $38.53 \pm 0.11$ & - & 40.00 & 0.99 & $5.0 \pm 0.7$ \\
\hline 49 & 09:32:13.32 & $21: 31: 21.74$ & 2.4 & 0 & 0 & $38.19 \pm 0.19$ & $2.72 \pm 0.09$ & 39.47 & 0.73 & $1.8 \pm 0.5$ \\
\hline 50 & 09:32:09.09 & $21: 28: 31.92$ & 3.03 & 0 & 0 & $38.42 \pm 0.18$ & - & 40.34 & 1.80 & $8.1 \pm 0.9$ \\
\hline 51 & $09: 32: 12.27$ & 21:30:08.09 & 2.7 & 0.03 & 83 & $38.21 \pm 0.23$ & - & 39.72 & 1.04 & $2.5 \pm 0.7$ \\
\hline 52 & 09:32:08.58 & 21:31:34.64 & 2.67 & 0 & 0 & $38.56 \pm 0.10$ & - & 40.10 & 1.09 & $5.9 \pm 0.7$ \\
\hline 53 & 09:32:11.28 & 21:31:16.48 & 2.5 & 0 & 0 & $38.17 \pm 0.21$ & $2.21 \pm 0.36$ & 39.93 & 1.48 & $3.4 \pm 0.6$ \\
\hline 54 & 09:32:12.06 & 21:31:11.01 & 3.67 & 0 & 0 & $38.53 \pm 0.20$ & $1.99 \pm 0.11$ & 40.29 & 1.47 & $7.8 \pm 1.2$ \\
\hline 55 & 09:32:10.77 & 21:29:56.08 & 2.5 & 0 & 0 & $38.27 \pm 0.17$ & $1.85 \pm 0.21$ & 40.29 & 2.00 & $6.9 \pm 0.6$ \\
\hline 56 & 09:32:11.01 & 21:30:03.02 & 3.2 & 0 & 0 & $38.51 \pm 0.16$ & - & 40.63 & 2.21 & $14.6 \pm 0.9$ \\
\hline 57 & $09: 32: 12.42$ & 21:29:30.64 & 3.17 & 0 & 0 & $38.47 \pm 0.17$ & - & 40.11 & 1.26 & $5.6 \pm 0.9$ \\
\hline 58 & 09:32:12.10 & 21:29:33.70 & 2.67 & 0 & 0 & $38.18 \pm 0.24$ & $3.15 \pm 0.07$ & 39.70 & 1.06 & $2.4 \pm 0.7$ \\
\hline 59 & 09:32:12.12 & 21:29:26.71 & 2.6 & 0 & 0 & $38.12 \pm 0.26$ & - & 39.78 & 1.30 & $2.6 \pm 0.6$ \\
\hline
\end{tabular}

The selection criteria for identifying a feature in the $\mathrm{H} \alpha$ image as an $\mathrm{H}$ II region is that the feature must have an area in pixels equal to or larger than the image spatial resolution (i.e.; area $\approx 32$ pixels; with a pixel size of $0.33^{\prime \prime} /$ pix), all pixels having an intensity of at least three times the r.m.s. noise above the local background intensity level. This selection criteria implies a detectability limit of $\sim 8.3 \times 10^{37} \mathrm{erg} \mathrm{s}^{-1}$. 
Table 2. continued.

\begin{tabular}{|c|c|c|c|c|c|c|c|c|c|c|}
\hline ID & $\begin{array}{c}\mathrm{RA} \\
(\mathrm{J} 2000)\end{array}$ & $\begin{array}{c}\text { Dec } \\
(\mathrm{J} 2000)\end{array}$ & $\begin{array}{l}\text { Radius } \\
(\operatorname{arcsec})\end{array}$ & $e$ & $\begin{array}{c}\text { PA } \\
(\mathrm{deg})\end{array}$ & $\begin{array}{l}\log L_{\mathrm{H}_{\alpha}+[\mathrm{NII}]} \\
(\text { in erg s} \\
\end{array}$ & $\begin{array}{c}\log E W_{\mathrm{H}_{\alpha}+[\mathrm{NII}]} \\
(\text { in } \AA \text { ) }\end{array}$ & $\begin{array}{c}\log \left(L_{24 \mu \mathrm{m}}\right) \\
\left(\operatorname{erg~s}^{-1}\right)\end{array}$ & $\begin{array}{c}A_{\mathrm{H} \alpha} \\
(\mathrm{mag})\end{array}$ & $\begin{array}{c}S F R \\
\left(10^{-3} M_{\odot} \mathrm{yr}^{-1}\right)\end{array}$ \\
\hline 60 & $09: 32: 12.21$ & $21: 29: 17.71$ & 2.43 & 0 & 0 & $38.06 \pm 0.26$ & - & 39.92 & 1.67 & $3.2 \pm 0.5$ \\
\hline 61 & 09:32:11.56 & $21: 29: 16.67$ & 3.2 & 0 & 0 & $38.32 \pm 0.25$ & $2.85 \pm 0.06$ & 40.03 & 1.38 & $4.4 \pm 0.9$ \\
\hline 62 & 09:32:10.49 & $21: 28: 57.76$ & 3.07 & 0.55 & 61 & $38.27 \pm 0.21$ & $2.29 \pm 0.05$ & 39.43 & 0.60 & $1.9 \pm 0.7$ \\
\hline 63 & $09: 32: 07.53$ & $21: 29: 19.29$ & 4.9 & 0.9 & -18 & $38.33 \pm 0.25$ & $2.46 \pm 0.04$ & 40.10 & 1.50 & $5.0 \pm 1.0$ \\
\hline 64 & 09:32:07.53 & 21:29:04.49 & 4.63 & 0.87 & 14 & $38.37 \pm 0.23$ & - & 40.16 & 1.54 & $5.8 \pm 1.0$ \\
\hline 65 & 09:32:08.01 & $21: 30: 28.55$ & 4.13 & 0 & 90 & $38.83 \pm 0.13$ & - & 39.96 & 0.56 & $6.7 \pm 1.6$ \\
\hline 66 & 09:32:08.18 & $21: 30: 42.83$ & 2.67 & 0 & 0 & $38.16 \pm 0.25$ & - & 39.09 & 0.39 & $1.2 \pm 0.7$ \\
\hline 67 & $09: 32: 12.63$ & $21: 29: 25.61$ & 2.33 & 0 & 0 & $38.13 \pm 0.21$ & - & 39.50 & 0.86 & $1.7 \pm 0.5$ \\
\hline
\end{tabular}

Notes. Column 1: ID number of the catalogued HiI regions; Cols. 2 and 3: equatorial coordinates; Col. 4: H II region radius in arcseconds; Cols. 5 and 6: eccentricity and position angle (measured North-West) of the integration apertures; Col. 7: decimal logarithm of the H $\alpha$ luminosity (in erg s${ }^{-1}$ ); Col. 8: decimal logarithm of the $\mathrm{H} \alpha$ equivalent width; Col. 9: decimal logarithm of the $24 \mu \mathrm{m}$ emission within the $\mathrm{H}$ II region apertures; Col. 10: $\mathrm{H} \alpha$ attenuation see Sect. 4.3; Col. 11: estimate of the current star-formation rate for each catalogued $\mathrm{H}$ II region. Columns 7-10 include the corresponding correction for Galactic extinction (Schlegel et al. 1998). The SFRs were obtained after applying a correction of $25 \%$ for [N II] contamination to the $\mathrm{H} \alpha$ luminosities and dust extinction as given in Col. 10.

A total of $67 \mathrm{H}$ II regions were catalogued and their positions, sizes, and luminosities were measured. We performed aperture photometry using GAIA ${ }^{4}$. Most of the catalogued regions are located in the s-shape region and its neighbourhood, a region which is closely related to the stellar bar. A representation of the catalogued H II regions can be seen in Fig. 3.

The H II region catalogue comprises the position, size, shape, and $\mathrm{H} \alpha+[\mathrm{N}$ II $]$ luminosity of the regions, and is presented in Table 2. The table contains the $\mathrm{H}$ II region identification number in Col. 1; the equatorial coordinates (Cols. 2 and 3). Column 4 shows the radius in arcseconds; Cols. 5 and 6 the eccentricity and position angle (measured northwest) of the integration aperture, and Col. 7 shows the decimal logarithm of the Galactic extinction-corrected $\mathrm{H} \alpha+[\mathrm{N}$ II] luminosity (not corrected for internal dust extinction).

\subsection{1. $\mathrm{H} \alpha$ equivalent width}

The $\mathrm{H} \alpha$ equivalent width (hereinafter $E W_{\mathrm{H} \alpha}$ ) is a measure of the strength of the $\mathrm{H} \alpha$ emission with respect to the continuum emission of the stellar ionizing cluster. The $E W_{\mathrm{H} \alpha}$ depends not only mainly on the evolutionary stage of the $\mathrm{H}$ II region (decreasing as the region ages), but also on the metallicity, the IMF, dust content, and ionizing photon leakage (e.g.; Bresolin \& Kennicutt 1999; Zurita \& Pérez 2008).

The $\mathrm{H} \alpha E W \mathrm{~s}$ were calculated from the ratio of our measured $\mathrm{H} \alpha$ luminosities (not corrected for internal dust extinction) to the continuum emission per $\AA$. The continuum emission of the ionizing cluster was estimated from the broad-band emission in the $R$-band at the position of the catalogued H II regions. Rather than using our Johnson $R$-band image of NGC 2903, we used the Sloan Digital Sky Survey (SDSS) $r^{\prime}$-band image. The latter has a higher spatial resolution than our image, and therefore allowed us to more clearly identify and measure the continuum cluster emission.

When computing the $\mathrm{H} \alpha E W$ of an $\mathrm{H}$ II region, we are interested only in the continuum radiation emitted by the ionizing cluster. However, both the flux from the ionizing cluster and the continuum emission from the underlying stellar population of the galaxy are included within the integration apertures. Correcting for this contamination is not straightforward and is

\footnotetext{
${ }^{4}$ GAIA is a derivative of the Skycat catalogue and image display tool, developed as part of the VLT project at ESO. Skycat and GAIA are free software under the terms of the GNU copyright.
}

the major source of uncertainty in $\mathrm{H}$ II region broad-band fluxes (Zurita \& Pérez 2008). We followed the procedure described in Zurita \& Pérez (2008) to estimate the contribution of the underlying stellar population to our measured broad-band fluxes by using two of the methods described there.

The first method consists of the measurement of the local background from the median value within annular apertures around the $\mathrm{H}$ II regions. The inner radius was set to be the effective radius of the $\mathrm{H}$ II region, and the annulus width to approximately three times the $\mathrm{HII}$ region radius.

The areas of the image corresponding to catalogued H II regions had previously been masked out in the image to avoid contamination within the annular apertures from neighbouring $\mathrm{H}$ II regions. For some regions, the median continuum emission within the annulus is clearly overestimated causing an underestimation of the cluster continuum emission, which sometimes even results in negative fluxes. This is generally due to the strong spatial variations in the disk continuum emission on small scales. Therefore, a second approach was adopted to more tightly constrain the local background estimates.

The second method uses growth curves and takes advantage of the continuum emission in most H II regions being less extended than the $\mathrm{H} \alpha$ emitting area. Therefore, the local background can be obtained from within the H II region area defined by its $\mathrm{H} \alpha$ emission. For each $\mathrm{H}$ II region, we derived radially averaged $r^{\prime}$-band surface-brightness profiles. For each region, we selected a radial range that was not contaminated by the ionizing cluster and determined the local background surface brightness from a fit to the profile in that range.

The $r^{\prime}$-band continuum fluxes were all corrected for the underlying stellar population contamination using both methods (when possible), giving us two estimates of the $r^{\prime}$-band flux of the ionizing cluster, from which we derived two values for the $E W_{\mathrm{H} \alpha+[\mathrm{NII}]}$. We calculated the difference between the two $E W_{\mathrm{H} \alpha+[\mathrm{NII}]}$ estimates of each $\mathrm{H}$ II region, and this was taken to be the measured $\mathrm{H} \alpha+[\mathrm{N}$ II $] E W$ uncertainty, so it represents the range of values covered when using the different estimates. Those regions for which the two estimates yielded differences larger than $600 \AA$, and those regions for which only one background measurement was available, were discarded, as we considered the results non-reliable. The resulting $\mathrm{H} \alpha E W$ s (including emission from the $\mathrm{N}$ II lines) are presented in Col. 8 of Table 2. 


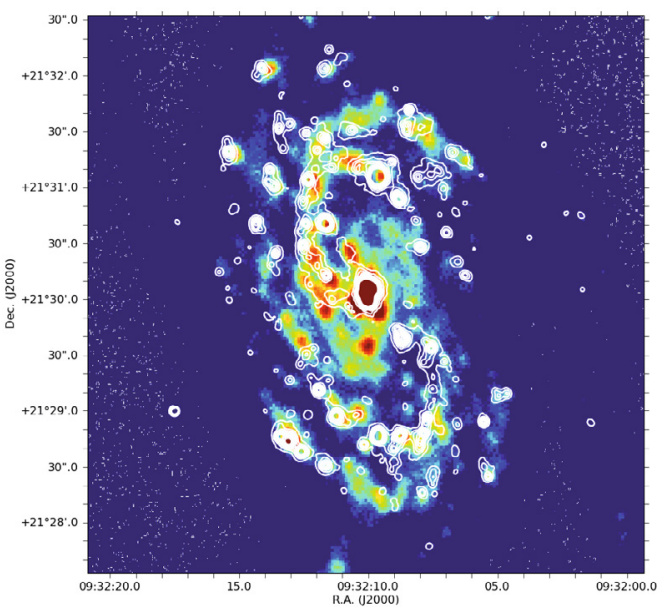

(a) NUV with overlaid $\mathrm{H} \alpha$ countours.

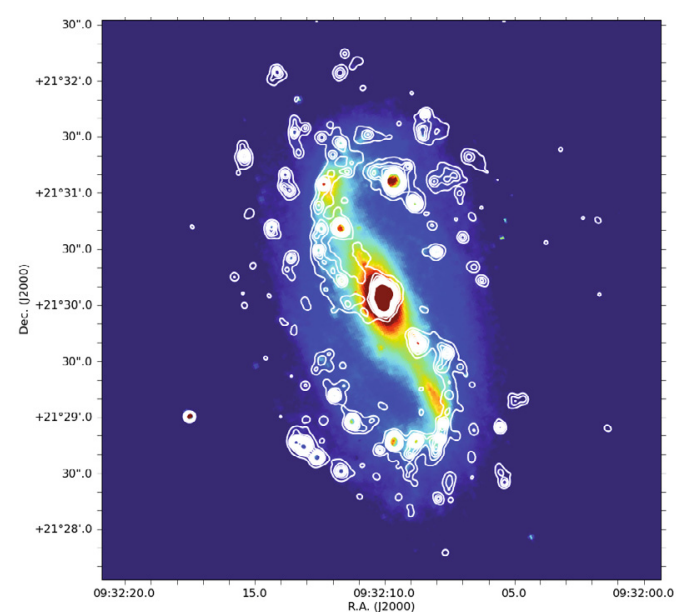

(c) $3.6 \mu \mathrm{m}$ with overlaid $\mathrm{H} \alpha$ contours.

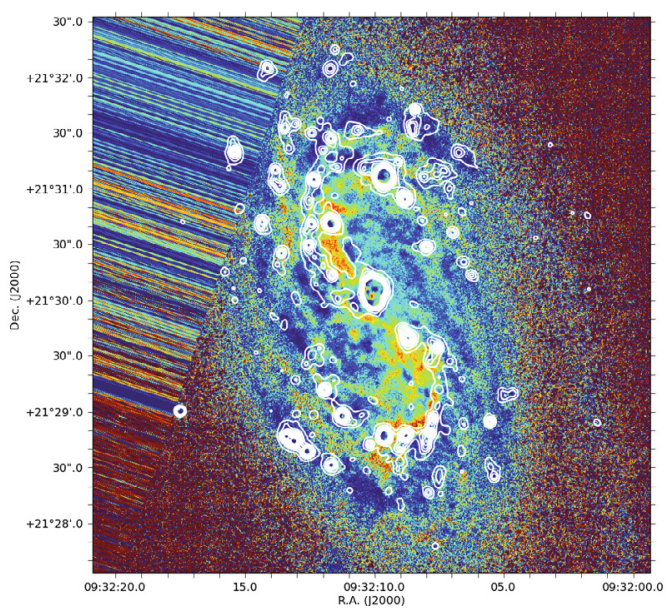

(e) $g^{\prime}-z^{\prime}$ colour map with overlaid $\mathrm{H} \alpha$ contours.

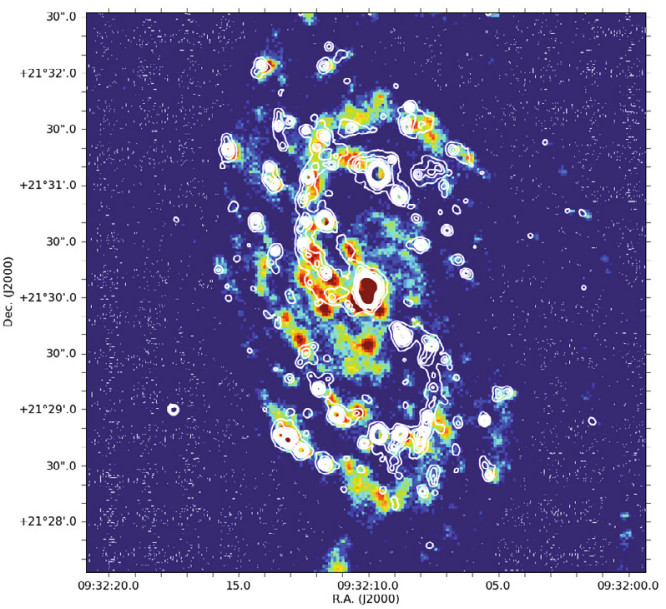

(b) FUV with overlaid $\mathrm{H} \alpha$ contours.

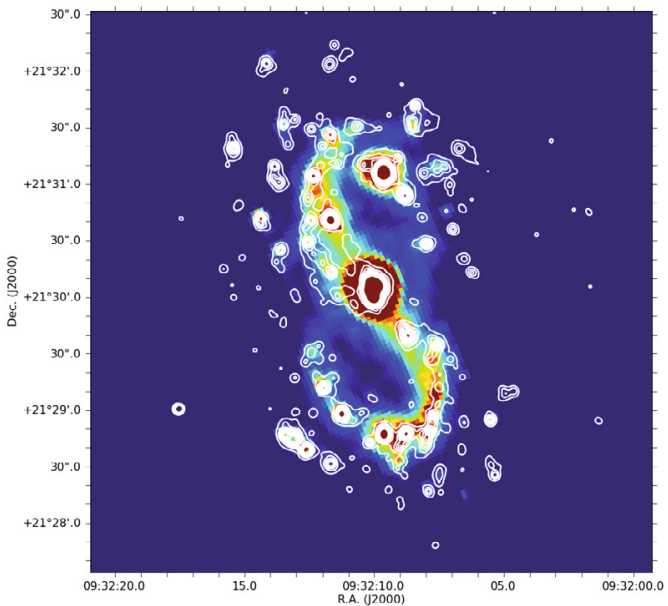

(d) $24 \mu \mathrm{m}$ with overlaid $\mathrm{H} \alpha$ contours.

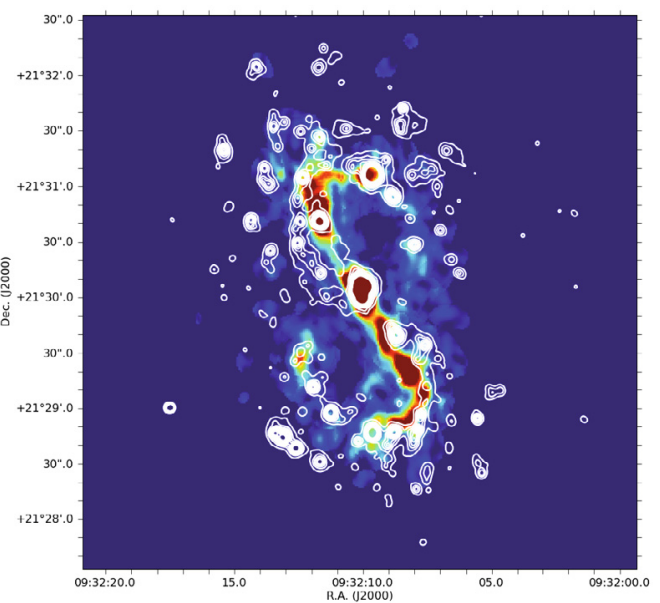

(f) $\mathrm{CO}(J=1-0)$ with overlaid $\mathrm{H} \alpha$ contours.

Fig. 2. Images of NGC 2903 in different spectral bands: a) GALEX NUV image, logarithmic scale b) GALEX FUV image, logarithmic scale c) Spitzer $3.6 \mu \mathrm{m}$ d) Spitzer $24 \mu \mathrm{m}$ e) $g^{\prime}-z^{\prime}$ colour-map f) CO $(J=1-0)$ transition map of NGC 2903. All the colour scales are given in arbitrary units. All images a) to f) show superimposed $\mathrm{H} \alpha$ contours, the resolution of each image is given in the text, Sect. 2. The $\mathrm{H} \alpha$ contour levels range between $4.5 \times 10^{35} \mathrm{erg} / \mathrm{s} / \mathrm{pix}$ and $4.5 \times 10^{36} \mathrm{erg} / \mathrm{s} / \mathrm{pix}$ (the $\mathrm{H} \alpha$ image pixel size is $0.33^{\prime \prime} / \mathrm{pix}$ ).

\subsection{UV photometry}

We performed aperture photometry on the NUV and FUV GALEX images to catalogue all the UV emitting knots of the bar region with the aim of obtaining their luminosities and colours. The aperture photometry was performed as described in Sect. 4.1 for the H II region catalogue, but using circular apertures centered on the FUV peaks. The location of the apertures is shown in Fig. 4. The same apertures and centres were used to measure fluxes on the $\mathrm{H} \alpha$ and $24 \mu \mathrm{m}$ with the 


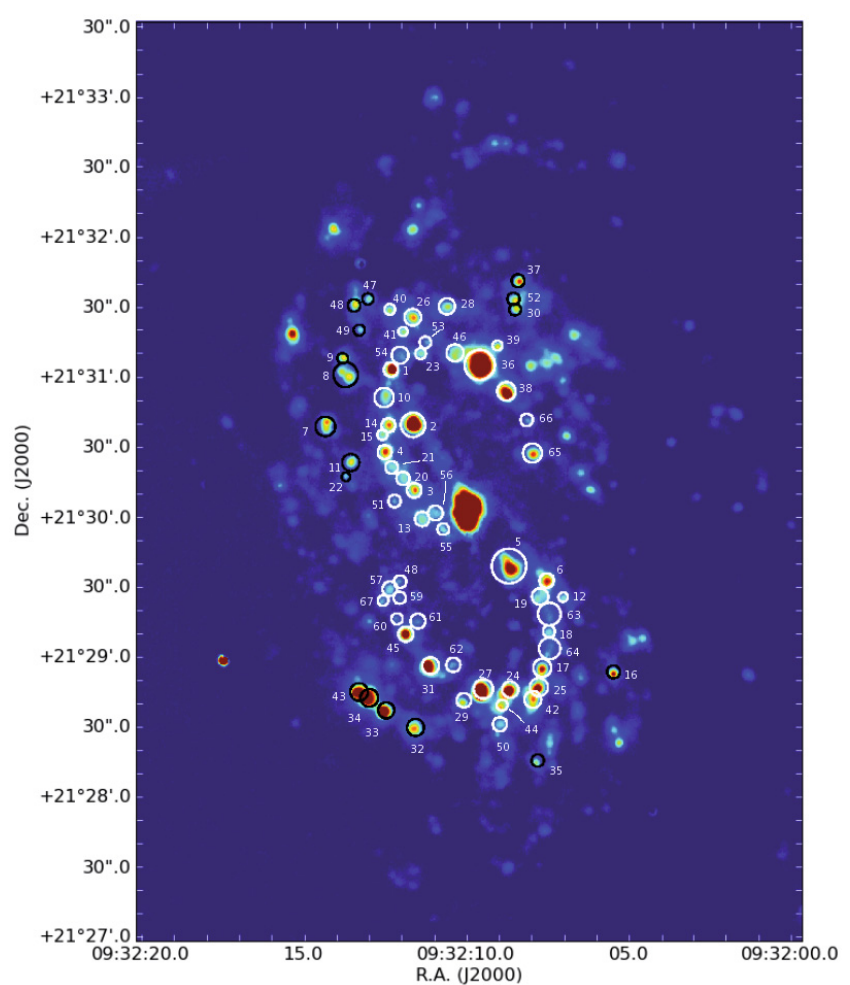

Fig. 3. H $\alpha$ continuum-subtracted image of NGC 2903. The white open circles indicate the position and size of the integration apertures of the catalogued H II regions belonging to the bar region. The black open circles indicate the $\mathrm{H}$ II regions closest to the catalogued UV complexes without $\mathrm{H} \alpha$ counterparts.

aim of deriving the dust attenuation (Sect. 4.3). The aperture size selection, 13.2 arcsec, corresponding to $\sim 570 \mathrm{pc}$ at the galaxy distance, was determined by the spatial resolution of the $24 \mu \mathrm{m}$ image and the emission peak shifts of the emitting knots at the different bands (see below in this section). These large apertures can cause some overlap between integration areas of neighbouring regions, but in none of the cases is the contamination significant.

As already mentioned for the $\mathrm{H} \alpha$ photometry in Sect. 4.1, the background emission has to be subtracted from the measured $24 \mu \mathrm{m}, \mathrm{NUV}$, and FUV fluxes within the aperture, to yield the stellar cluster emission alone. When estimating the background contamination, the annuli around each aperture are difficult to define because of crowding. As an alternative method, we fitted light profiles perpendicular to the bar major axis averaged over a rectangle of width $\approx 40^{\prime \prime}$ (much larger than our photometric apertures for averaging out small-scale background variations, but small enough to ensure a valid local background estimate). The local background of each region was then obtained by interactive interpolation of the background on the corresponding fitted profile (second order polynomial) at the position of the emission peaks. A total of 56 regions were catalogued in the bar region alone (excluding the galaxy centre) and the positions, luminosities, and UV colours are shown in Table 3.

The peaks of emission in the UV and the $24 \mu \mathrm{m}$ (and $\mathrm{H} \alpha$ ) image are offset from each other by a few arcseconds in most of the regions. This offset between the emission peaks in UV and $24 \mu \mathrm{m}$ (and $\mathrm{H} \alpha$ ) amounts to $6^{\prime \prime}$ and was already noticed by Calzetti et al. (2005) for M 51.

As already said, the $24 \mu \mathrm{m}$ and $\mathrm{H} \alpha$ emission peaks do not always fall within the UV apertures. Therefore, the $\mathrm{H} \alpha$ and $24 \mu \mathrm{m}$

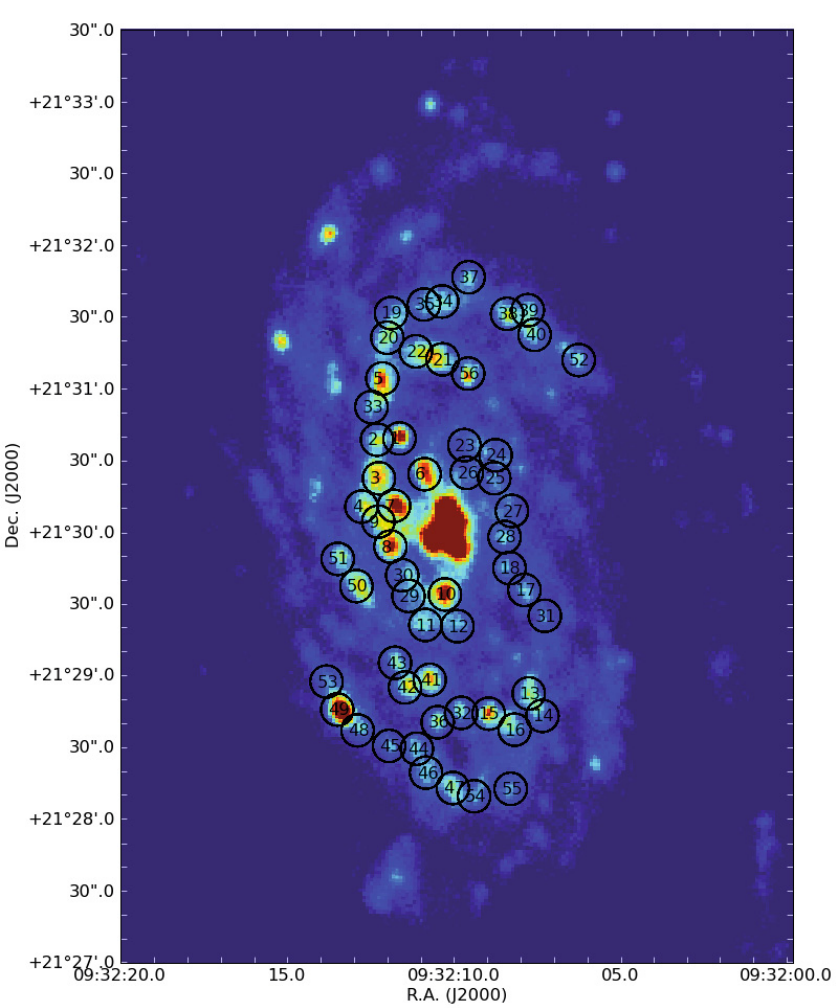

Fig. 4. GALEX NUV image of NGC 2903 with the apertures used for photometry in the FUV, NUV, $\mathrm{H} \alpha$, and $24 \mu$ m images. See Sect. 4.2 for further details.

luminosities shown in Table 3 should be used with care, as they represent the luminosities of regions centered on the UV peaks with the unique aim of obtaining the dust attenuation in the UV. For the $\mathrm{H} \alpha$ luminosities of the $\mathrm{H}$ II regions, we refer the reader to Sect. 4.1 and Table 2. Last column of Table 3 shows the ID number of the $\mathrm{H}$ II region catalogue, which matches the UV emitting knot.

A careful visual inspection of the UV, the $\mathrm{H} \alpha$, and the $24 \mu \mathrm{m}$ images of NGC 2903 indicates that a number of compact bright UV emission knots, mainly located in the bar region, in the southeastern and northwestern area from the nucleus, have significant counterpart emission at neither $\mathrm{H} \alpha$ nor $24 \mu \mathrm{m}$ wavelengths (see Fig. 2).

\subsection{Dust attenuation}

For many years, much effort has gone into deriving reliable and easy-to-use dust extinction indicators to correct optical and UV observations (e.g.; Kennicutt 1983; Calzetti et al. 2000; Iglesias-Páramo et al. 2006). The appearance of multiwavelength observations from the FUV to the mid-infrared of a relatively large sample of nearby galaxies (e.g.; SINGS; Kennicutt et al. 2003) allowed us to determine a reliable extinction correction using all the available bands. The $\mathrm{H} \alpha$ and $24 \mu \mathrm{m}$ combination has been shown to be a very useful tool for estimating attenuation-corrected $\mathrm{H} \alpha$ and UV fluxes (Kennicutt et al. 2007, 2009). We used these results to obtain dust-corrected UV fluxes. We calculated the attenuation in the UV bands from the $\mathrm{H} \alpha$ attenuation using the laws presented by Calzetti (2001)

$A_{0.16, \mathrm{star}}=1.78 A_{\mathrm{H} \alpha, \mathrm{gas}} \quad(\mathrm{FUV})$
$A_{0.28, \mathrm{star}}=1.29 A_{\mathrm{H} \alpha, \mathrm{gas}} \quad(\mathrm{NUV})$, 
Table 3. Summary of positions and photometry of the star-forming knots.

\begin{tabular}{|c|c|c|c|c|c|c|c|c|c|c|c|}
\hline$\overline{\mathrm{ID}}$ & $\begin{array}{l}\text { RA } \\
\text { (J2000) }\end{array}$ & $\begin{array}{l}\begin{array}{l}\text { Dec } \\
(\mathrm{J} 2000)\end{array}\end{array}$ & $\begin{array}{l}\log L_{\mathrm{H} \alpha} \\
\left(\mathrm{erg} \mathrm{s}^{-1}\right)\end{array}$ & $\begin{array}{l}\log L_{24 \mu \mathrm{m}} \\
\left(\mathrm{erg} \mathrm{s}^{-1}\right)\end{array}$ & $\begin{array}{l}\log L_{\mathrm{FUV}} \\
\left(\mathrm{erg} \mathrm{s}^{-1}\right)\end{array}$ & $\begin{array}{l}\log L_{\mathrm{NUV}} \\
\left(\mathrm{erg} \mathrm{s}^{-1}\right)\end{array}$ & $\begin{array}{l}\begin{array}{l}A_{\mathrm{H} \alpha} \\
(\mathrm{mag})\end{array} \\
\end{array}$ & $\begin{array}{l}\begin{array}{l}\text { FUV } \\
(\mathrm{mag})\end{array} \\
\end{array}$ & $\begin{array}{l}\begin{array}{l}\text { NUV } \\
(\mathrm{mag})\end{array} \\
\end{array}$ & $\begin{array}{l}\begin{array}{l}\text { FUV-NUV } \\
(\mathrm{mag})\end{array} \\
\end{array}$ & 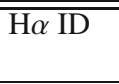 \\
\hline 1 & $09: 32: 11.67$ & $21: 30: 40.80$ & 39.15 & 40.92 & 40.63 & 40.73 & 1.27 & 15.74 & 15.67 & 0.08 & 2 \\
\hline 2 & $09: 32: 12.34$ & $21: 30: 40.00$ & 38.93 & 40.57 & 40.53 & 40.61 & 1.06 & 16.37 & 16.22 & 0.15 & 14,15 \\
\hline 3 & $09: 32: 12.25$ & $21: 30: 23.80$ & 38.87 & 40.32 & 40.80 & 40.83 & 0.78 & 16.19 & 16.03 & 0.16 & $4,20,21$ \\
\hline 4 & $09: 32: 12.75$ & $21: 30: 11.70$ & $<38.48$ & $<39.41$ & 40.65 & 40.58 & 0 & 17.95 & 17.68 & 0.27 & - \\
\hline 5 & $09: 32: 12.23$ & $21: 31: 05.70$ & 38.94 & 40.71 & 40.74 & 40.81 & 1.29 & 15.44 & 15.45 & -0.01 & 1 \\
\hline 6 & 09:32:10.89 & $21: 30: 26.10$ & 38.65 & 40.47 & 40.69 & 40.83 & 1.36 & 15.44 & 15.30 & 0.14 & - \\
\hline 7 & 09:32:11.78 & $21: 30: 12.50$ & 38.86 & 40.32 & 40.90 & 40.91 & 0.8 & 15.89 & 15.81 & 0.08 & 2,3 \\
\hline 8 & $09: 32: 11.85$ & $21: 29: 55.30$ & $<38.48$ & $<39.53$ & 40.77 & 40.80 & 0 & 17.65 & 17.13 & 0.52 & 13 \\
\hline 9 & 09:32:12.23 & 21:30:05.70 & $<38.48$ & $<39.75$ & 40.71 & 40.74 & 0 & 17.79 & 17.26 & 0.53 & 51 \\
\hline 10 & 09:32:10.18 & $21: 29: 35.90$ & $<38.48$ & $<39.82$ & 40.79 & 40.87 & 0 & 17.59 & 16.95 & 0.64 & - \\
\hline 11 & $09: 32: 10.74$ & $21: 29: 22.90$ & $<38.48$ & $<38.94$ & 40.41 & 40.54 & 0 & 18.55 & 17.78 & 0.77 & - \\
\hline 12 & 09:32:09.78 & $21: 29: 22.80$ & $<38.48$ & $<39.53$ & 40.32 & 40.41 & 0 & 18.77 & 18.10 & 0.67 & - \\
\hline 13 & 09:32:07.59 & $21: 28: 55.40$ & 38.97 & 40.64 & 40.52 & 40.64 & 1.11 & 16.29 & 16.08 & 0.21 & 17 \\
\hline 14 & 09:32:07.16 & $21: 28: 46.10$ & 38.75 & 40.03 & 40.43 & 40.46 & 0.6 & 17.45 & 17.21 & 0.23 & 25 \\
\hline 15 & 09:32:08.77 & $21: 28: 46.40$ & 39.17 & 40.84 & 40.64 & 40.71 & 1.1 & 16.00 & 15.91 & 0.09 & 24 \\
\hline 16 & 09:32:07.98 & $21: 28: 40.00$ & 38.88 & 40.51 & 40.54 & 40.59 & 1.06 & 16.35 & 16.29 & 0.06 & 42 \\
\hline 17 & 09:32:07.81 & $21: 29: 38.70$ & 38.90 & 40.38 & 40.09 & 40.23 & 0.83 & 17.88 & 17.49 & 0.39 & - \\
\hline 18 & 09:32:08.27 & $21: 29: 47.70$ & 38.87 & 40.26 & 40.05 & 40.14 & 0.71 & 18.18 & 17.86 & 0.33 & - \\
\hline 19 & 09:32:12.03 & $21: 31: 33.30$ & 38.70 & 40.34 & 40.54 & 40.58 & 1.07 & 16.31 & 16.31 & 0.01 & 26 \\
\hline 20 & $09: 32: 12.12$ & $21: 31: 22.90$ & 38.70 & 40.49 & 40.64 & 40.68 & 1.32 & 15.63 & 15.72 & -0.1 & 41 \\
\hline 21 & $09: 32: 10.45$ & $21: 31: 14.50$ & 38.83 & 40.70 & 40.65 & 40.75 & 1.45 & 15.36 & 15.38 & -0.03 & 46 \\
\hline 22 & $09: 32: 11.25$ & $21: 31: 17.50$ & 38.78 & 40.39 & 40.62 & 40.74 & 1.02 & 16.20 & 15.95 & 0.25 & 23 \\
\hline 23 & 09:32:09.72 & $21: 30: 38.80$ & $<38.48$ & $<39.53$ & 40.19 & 40.34 & 0 & 19.10 & 18.27 & 0.82 & - \\
\hline 24 & 09:32:08.78 & $21: 30: 34.90$ & $<38.48$ & $<39.84$ & 40.26 & 40.27 & 0 & 18.91 & 18.44 & 0.48 & - \\
\hline 25 & 09:32:08.80 & $21: 30: 25.20$ & $<38.48$ & $<39.94$ & 40.17 & 40.20 & 0 & 19.15 & 18.63 & 0.52 & - \\
\hline 26 & 09:32:09.63 & $21: 30: 27.20$ & $<38.48$ & $<40.07$ & 40.12 & 40.27 & 0 & 19.28 & 18.44 & 0.84 & - \\
\hline 27 & 09:32:08.25 & $21: 30: 11.70$ & $<38.48$ & $<39.85$ & 40.09 & 40.06 & 0 & 19.35 & 18.96 & 0.39 & - \\
\hline 28 & 09:32:08.45 & 21:30:00.70 & $<38.48$ & $<40.06$ & 40.17 & 40.20 & 0 & 19.16 & 18.61 & 0.55 & - \\
\hline 29 & $09: 32: 11.27$ & $21: 29: 34.90$ & $<38.48$ & $<39.52$ & 40.37 & 40.44 & 0 & 18.64 & 18.02 & 0.62 & - \\
\hline 30 & 09:32:11.47 & $21: 29: 43.50$ & $<38.48$ & $<39.81$ & 40.39 & 40.36 & 0 & 18.59 & 18.22 & 0.37 & - \\
\hline 31 & 09:32:07.17 & $21: 29: 28.20$ & 38.83 & 40.24 & 39.79 & 39.94 & 0.74 & 18.77 & 18.31 & 0.46 & 6 \\
\hline 32 & 09:32:09.60 & $21: 28: 46.50$ & 39.16 & 41.03 & 40.37 & 40.44 & 1.45 & 16.06 & 16.16 & -0.09 & 27 \\
\hline 33 & 09:32:12.54 & $21: 30: 53.60$ & 38.76 & 40.63 & 40.45 & 40.59 & 1.46 & 15.84 & 15.75 & 0.09 & 10 \\
\hline 34 & 09:32:10.51 & $21: 31: 38.80$ & $<38.48$ & $<40.07$ & 40.58 & 40.64 & 0 & 18.12 & 17.54 & 0.59 & 28 \\
\hline 35 & 09:32:11.05 & $21: 31: 37.20$ & $<38.48$ & $<40.19$ & 40.54 & 40.58 & 0 & 18.22 & 17.67 & 0.56 & - \\
\hline 36 & $09: 32: 10.29$ & $21: 28: 42.20$ & 38.62 & 40.53 & 40.40 & 40.47 & 1.53 & 15.84 & 15.98 & -0.14 & 29 \\
\hline 37 & 09:32:09.74 & $21: 31: 49.20$ & $<38.48$ & $<39.48$ & 40.42 & 40.49 & 0 & 18.52 & 17.89 & 0.63 & - \\
\hline 38 & 09:32:08.56 & $21: 31: 34.30$ & 38.76 & 40.44 & 40.61 & 40.65 & 1.12 & 16.05 & 16.06 & -0.01 & 30,52 \\
\hline 39 & 09:32:07.94 & $21: 31: 36.10$ & 38.82 & 40.01 & 40.59 & 40.61 & 0.5 & 17.20 & 16.97 & 0.23 & 37 \\
\hline 40 & 09:32:07.72 & $21: 31: 25.80$ & $<38.48$ & $<39.31$ & 40.54 & 40.60 & 0 & 18.22 & 17.63 & 0.59 & - \\
\hline 41 & $09: 32: 10.56$ & $21: 28: 59.90$ & 38.92 & 40.38 & 40.67 & 40.72 & 0.81 & 16.48 & 16.28 & 0.19 & - \\
\hline 42 & $09: 32: 11.30$ & $21: 28: 56.40$ & 38.90 & 40.76 & 40.64 & 40.66 & 1.42 & 15.46 & 15.64 & -0.18 & 31 \\
\hline 43 & $09: 32: 11.61$ & $21: 29: 06.70$ & 38.75 & 40.52 & 40.47 & 40.51 & 1.27 & 16.15 & 16.20 & -0.05 & 45 \\
\hline 44 & $09: 32: 10.89$ & $21: 28: 30.90$ & $<38.48$ & $<40.03$ & 40.52 & 40.52 & 0 & 18.28 & 17.82 & 0.47 & - \\
\hline 45 & 09:32:11.72 & $21: 28: 31.70$ & 38.74 & 40.16 & 40.39 & 40.36 & 0.76 & 17.26 & 17.24 & 0.01 & 32 \\
\hline 46 & 09:32:10.59 & $21: 28: 21.00$ & $<38.48$ & $<39.53$ & 40.55 & 40.57 & 0 & 18.20 & 17.71 & 0.49 & - \\
\hline 47 & 09:32:09.78 & $21: 28: 14.80$ & $<38.48$ & $<39.74$ & 40.64 & 40.60 & 0 & 17.97 & 17.61 & 0.36 & - \\
\hline 48 & $09: 32: 12.68$ & $21: 28: 37.90$ & 39.01 & 40.57 & 40.54 & 40.53 & 0.94 & 16.55 & 16.59 & -0.04 & 33 \\
\hline 49 & $09: 32: 13.30$ & $21: 28: 46.40$ & 39.07 & 40.46 & 41.10 & 41.04 & 0.72 & 15.54 & 15.60 & -0.06 & 34,43 \\
\hline 50 & $09: 32: 12.83$ & $21: 29: 38.40$ & 38.48 & 39.71 & 40.66 & 40.59 & 0 & 17.92 & 17.64 & 0.28 & - \\
\hline 51 & $09: 32: 13.41$ & $21: 29: 49.60$ & $<38.48$ & $<39.63$ & 40.40 & 40.33 & 0 & 18.57 & 18.29 & 0.28 & - \\
\hline 52 & 09:32:06.38 & $21: 31: 15.80$ & 38.65 & 39.77 & 40.42 & 40.44 & 0.43 & 17.75 & 17.47 & 0.28 & - \\
\hline 53 & $09: 32: 13.65$ & $21: 28: 58.00$ & $<38.48$ & $<39.77$ & 40.47 & 40.49 & 0 & 18.39 & 17.90 & 0.49 & - \\
\hline 54 & 09:32:09.14 & $21: 28: 11.60$ & $<38.48$ & $<39.98$ & 40.48 & 40.47 & 0 & 18.38 & 17.95 & 0.43 & - \\
\hline 55 & 09:32:08.05 & $21: 28: 15.20$ & $<38.48$ & $<40.14$ & 40.30 & 40.40 & 0 & 18.82 & 18.11 & 0.71 & 35 \\
\hline 56 & 09:32:09.67 & 21:31:08.80 & 39.42 & 41.32 & 40.25 & 40.44 & 1.5 & 16.26 & 16.08 & 0.18 & 36 \\
\hline
\end{tabular}

Notes. Column 1: ID of the regions; Cols. 2 and 3: right ascension and declination respectively; Cols. 4-7: decimal logarithm of the H $\alpha, 24 \mu \mathrm{m}$ FUV and NUV luminosities respectively, these values have not been corrected for extinction; Col. 8: estimated $\mathrm{H} \alpha$ attenuation in magnitudes; Cols. 9 and 10: FUV and NUV attenuation-corrected magnitudes; Col. 11: the FUV-NUV colour of the star forming region. Column 4 shows luminosities corrected for a $25 \%$ [N II] contamination. For the regions where Col. 8 has a value of 0.0, Cols. 4 and 5 correspond to an upper limit of the corresponding luminosities. 


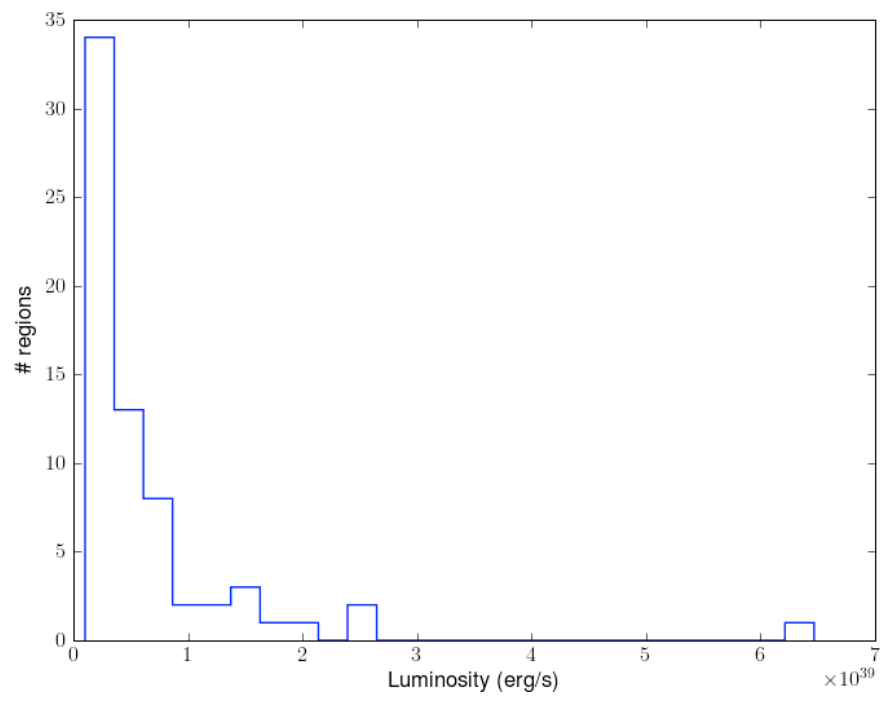

Fig. 5. Distribution of the $\mathrm{H} \alpha+[\mathrm{N}$ II $]$ luminosity of the catalogued $\mathrm{H}$ II regions across the bar zone of NGC 2903 from which the $E W_{\mathrm{H} \alpha}$ were calculated.

where $A_{\mathrm{X}}$ is the attenuation, in magnitudes, at the specific band. To obtain the $\mathrm{H} \alpha$ attenuation, we used the method described by Kennicutt et al. (2007)

$A_{\mathrm{H} \alpha}=2.5 \log \left[1+\frac{a L_{24} \mu \mathrm{m}}{\left(L_{\mathrm{H} \alpha}\right)_{\mathrm{obs}}}\right]$,

where $\left(L_{\mathrm{H} \alpha}\right)_{\text {obs }}$ refers to the observed $\mathrm{H} \alpha$ luminosity with correction for the $\mathrm{N}$ II lines, $L_{24 \mu \mathrm{m}}$ is defined as the product $v L_{v}$, and $a$ is the $L_{24 \mu \mathrm{m}} / L_{\mathrm{H} \alpha}$ constant scaling factor, which is fitted empirically. A value of 0.038 was adopted for $a$, as derived in the same paper for the derivation of individual region parameters, and a value of $a=0.02$ was adopted when deriving galaxy integrated parameters. Galactic extinction was applied to the calculation of the dust attenuation. Errors in the attenuation and UV magnitudes follow from two uncertainties; first, the photometric calibration uncertainties and second, the local background estimates. Errors are of the order of $10 \%$.

Because no dust tracers are detected in the UV emitting regions without $\mathrm{H} \alpha$ and $24 \mu \mathrm{m}$ emission counterparts, we adopted a value of zero for the attenuation in these regions. Besides the lack of $\mathrm{H} \alpha$ and $24 \mu \mathrm{m}$, neither $8 \mu \mathrm{m}$ (Dale et al. 2009) nor CO $(J=1-0)$ emission is detected in these regions, indicating that there is very little gas or dust obscuring the UV emission. As an $L_{\mathrm{H} \alpha}$ upper limit for these regions we assumed $L_{\mathrm{H} \alpha+[\mathrm{NII}]}=$ $3.8 \times 10^{38} \mathrm{erg} \mathrm{s}^{-1}$, which is the integrated $\mathrm{H} \alpha$ luminosity in a 13.2 arcsec aperture assuming a constant flux per pixel equal to the rms noise of the $\mathrm{H} \alpha$ image background. The derived $\mathrm{H} \alpha$ attenuations for the complete catalogue are presented in Table 3. We then derived UV-corrected magnitudes and calculated the FUV-NUV colour for each region. These results are also presented in Table 3: Col. 1 indicates the ID of the regions; Cols. 2 and 3 the equatorial coordinates; Cols. 4-7 the $\mathrm{H} \alpha, 24 \mu \mathrm{m}, \mathrm{FUV}$, and NUV luminosities, respectively; Col. 8 the $\mathrm{H} \alpha$ attenuation; Cols. 9 and 10 the FUV and NUV attenuation-corrected magnitudes, respectively; Col. 11 the FUV-NUV colour derived; and Col. 12 the $\mathrm{H}$ II region ID (taken from Table 2) located close to each of the corresponding UV knot.

\section{Results}

\section{1. $H_{\alpha}$ luminosity and $E W_{H \alpha}$}

The logarithms of the $\mathrm{H} \alpha+[\mathrm{NII}]$ luminosities of the HII regions of NGC 2903 range from 38.0 to $39.8 \mathrm{erg} \mathrm{s}^{-1}$ and their distribution is shown in a histogram in Fig. 5. The luminosities are within the range of typical values for extragalactic $\mathrm{H}$ II regions in barred and unbarred galaxies (see e.g.; Kennicutt et al. 1989; Mayya 1994; González-Delgado \& Pérez 1997; Rozas et al. 1999; Relaño et al. 2005), but are higher than the H $\alpha$ luminosities reported by Alonso-Herrero et al. (2001) for the nuclear region of NGC 2903. This apparent discrepancy is partly caused by the smaller distance assumed for NGC 2903 by these authors (6.3 Mpc versus 8.9 Mpc assumed in this paper). More importantly, Alonso-Herrero et al. (2001) apply a semi-automated method based on a limiting $\mathrm{H} \alpha$ surface brightness to define the edges of an H II region from an HS T image, yielding catalogued $\mathrm{H} \alpha$ knots that may correspond to parts of a single $\mathrm{H}$ II region, as defined in ground-based $\mathrm{H} \alpha$ imaging, and therefore have lower luminosities and sizes.

The H II region number 36, located in the northernmost part of the bar (see Table 2 and/or Fig. 3), has an $\mathrm{H} \alpha$ luminosity of $6.4 \times 10^{39} \mathrm{erg} \mathrm{s}^{-1}$, which makes this region the brightest $\mathrm{H}$ II region in the galaxy (outside the nuclear region of the galaxy). Its luminosity is comparable to, for instance the brightest $\mathrm{H}$ II region in the nearby spiral galaxy M 51 (Calzetti et al. 2005; Scoville et al. 2001) and 30 Doradus in the Large Magellanic Cloud (Kennicutt et al. 1989).

The $E W_{\mathrm{H} \alpha+[\mathrm{NII}]}$ of $\mathrm{H}$ II regions in the bar of NGC 2903 range from $\sim 71$ to $\sim 1550 \AA$, which is typical of disk and bar H II regions (e.g.; Bresolin \& Kennicutt 1999; von Hippel \& Bothum 1990; Cedrés et al. 2005; Zurita \& Pérez 2008). The distribution of $E W_{\mathrm{H} \alpha+[\mathrm{NII}]}$ has a mean value of $\log E W_{\mathrm{H} \alpha+[\mathrm{NII}]}=2.6 \pm 0.3$ $\left(E W_{\mathrm{H} \alpha}\right.$ in $\AA$ ).

The $\mathrm{H} \alpha$ equivalent width of $\mathrm{H}$ II regions depends on several factors including the initial mass function (IMF), the evolutionary status of the HII region, its metal content, and ionizing photon loss due to dust extinction or leakage from the region. Zurita \& Pérez (2008) analysed the effect of all these factors on the observed distribution of $E W_{\mathrm{H} \alpha}$ for the bar H II regions in NGC 1530, and we refer the reader to that paper for a more detailed discussion of the subject. Assuming similar physical conditions in the bar of NGC 2903, the evolutionary status of the $\mathrm{H}$ II region is the dominant factor affecting the $E W_{\mathrm{H} \alpha}$ distribution. Assuming also that the $\mathrm{H}$ II region is the product of a single burst of star-formation, population synthesis models such as Starburst99 (Leitherer et al. 1999) can be used to estimate an average age for each H II region, for a given IMF and metallicity. We therefore compared the theoretical predictions of Starburst 99 for the evolution of a single burst of star-formation with age, with our $E W_{\mathrm{H} \alpha}$ measurements. Our Starburst 99 simulations used the Geneva stellar-evolutionary tracks, and a metallicity of $Z=0.02$ was assumed, which is the mean oxygen abundance of the gas in NGC 2903 at the range of galactocentric distances covered by the NGC 2903 bar (Pilyugin et al. 2004). For the IMF, we assumed a multi power-law parametrization $\left(\mathrm{d} N / \mathrm{d} m \propto m^{-\alpha}\right)$ with $\alpha=1.35$ for $0.1<M<0.5 M_{\odot}$ and $\alpha=2.35$ (Salpeter) for higher masses up to $100 M_{\odot}$. From the comparison of the Starburst 99 output with our measurements (after correcting for [N II] contamination), we estimate that the ages of the catalogued H II regions of NGC 2903 are between $2.7 \mathrm{Myr}\left(\log E W_{\mathrm{H}_{\alpha}}=3.07\right)$ and 7.1 Myr $\left(\log E W_{\mathrm{H}_{\alpha}}=1.73\right)$. We note that to derive an age from the measured equivalent 
width, we first had to correct the measured equivalent width of the $[\mathrm{N}$ II] lines contribution.

Figure 6 shows the distribution of the $E W_{\mathrm{H}_{\alpha}}$ in the galaxy on top of $3.6 \mu \mathrm{m}$ intensity contours. As mentioned earlier, the $3.6 \mu \mathrm{m}$ emission is a strong tracer of old underlying stellar populations and therefore a good indicator of the stellar bar. The distribution of the $E W_{\mathrm{H}_{\alpha}}$ shows no correlation with position within the bar.

\subsection{Dust attenuation}

Figure 8 presents the distribution and amplitude of the $\mathrm{H} \alpha$ attenuation in the bar of NGC 2903 with the $3.6 \mu$ m intensity contours (tracing the stellar bar) overlaid on top. The attenuation was calculated from the ratio of the $\mathrm{H} \alpha$ to the $24 \mu \mathrm{m}$ emission (Kennicutt et al. 2007) as presented in Sect. 4.3. The apertures are centered on regions of strong UV emission.

The mean attenuation value is around $A_{\mathrm{H} \alpha} \sim 1.0 \mathrm{mag}$ and ranges from 0 to $1.5 \mathrm{mag}$ (except for regions 55 and 56, closer to the nucleus with an attenuation value of 2.2). There are a few UV regions where we assume no dust attenuation because of a lack of any significant $\mathrm{H} \alpha$ and $24 \mu \mathrm{m}$ emission (see Sect. 4.3). The higher attenuation values are located in the bar and at the beginning of the spiral pattern (see Table 3). This indicates that the bar and spiral arms in NGC 2903 are gas rich, whereas the UV emitting regions to the northwest and southeast of the galaxy nucleus, those regions with zero attenuation, have low gas and dust content; nevertheless, this region is populated by young stellar clusters, see Sect. 5.4. Additional discussion of the origin of these regions is presented in Sect. 6 .

\subsection{Star-formation rate}

The locations of the most active star-formation sites within a bar are determined by the favoured dynamical behaviour of the gas and dust. Studying the distribution of the loci of current starformation within bars can therefore put constraints on the properties of a particular bar potential. Gas does not stay in typical bar intersecting orbits and tends to accumulate and shock in certain regions of the bar. Dust lanes can be used as tracers of shocks in the gas flow (Athanassoula 1992). From Fig. 2, one can already see that the dust lanes (as seen from the $g^{\prime}-z^{\prime}$ colour map) follow the $\mathrm{CO}(J=1-0)$ distribution, the $\mathrm{H} \alpha$ leading the $\mathrm{CO}(J=1-0)$ as previously observed in other barred spirals (e.g.; Sheth et al. 2002). The $\mathrm{H} \alpha$ emission in the bar of NGC 2903 follows an s-shape covering the whole stellar bar and is dominated by classical localised H II regions (Fig. 3).

In ionization-bounded $\mathrm{H}$ II regions, the reddening-corrected $\mathrm{H} \alpha$ luminosity scales directly with the total Lyman continuum flux emitted by the ionizing embedded star cluster. Therefore, the $\mathrm{H} \alpha$ emission is a good tracer of current star-formation. We used the following calibration relating the $\mathrm{H} \alpha$ luminosity to the rate of star-formation (Kennicutt 1998)

$\operatorname{SFR}_{\mathrm{H} \alpha}\left(M_{\odot} \operatorname{year}^{-1}\right)=7.9 \times 10^{-42} L_{\mathrm{H} \alpha}\left(\mathrm{erg} \mathrm{s}^{-1}\right)$,

for calculating the current SFR associated with each catalogued H II region (Sect. 4.1). The results are given in Col. 11 of Table 2 and were obtained from the observed $\mathrm{H} \alpha$ luminosities (Col. 4 of Table 2), and were corrected for [N II] emissionlines contamination (see Sect. 2.1) and dust extinction inside the regions. The latter were estimated as already described in Sect. 4.3 for the UV emitting knots, comparing the $\mathrm{H} \alpha$ and $24 \mu \mathrm{m}$ luminosities within the H II region apertures. Given the

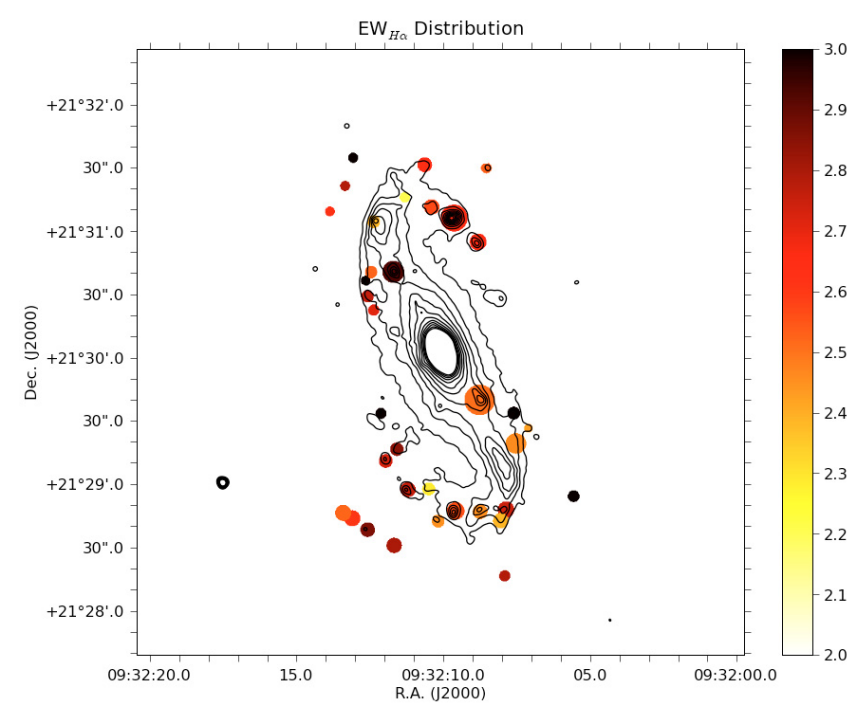

Fig. 6. $\mathrm{H} \alpha+[\mathrm{N}$ II $] E W$ distribution in the bar region of NGC 2903. The size of the circles represents the $\mathrm{H}$ II region projected area. The intensity contours trace the $3.6 \mu \mathrm{m}$ emission.

different spatial resolution of the two images, we applied an aperture correction factor (Table 1 of Engelbracht et al. 2007). The SFRs obtained for our catalogued H II regions range from $\sim 1.1$ to $\sim 126 \times 10^{-3} M_{\odot}$ year $^{-1}$. We excluded regions 55 and 56 in this calculation because of likely contamination from the nuclear region emission.

The total SFR implied by the $\mathrm{H} \alpha$ emission of the bar zone of NGC 2903 (excluding the nucleus) is $\sim 0.9 \pm 0.2 M_{\odot} \mathrm{yr}^{-1}$ (Fig. 7 shows the integration area). This value implies that the NGC 2903 bar is forming stars at a rate comparable to other normal (non-starburst) spirals. The nucleus of NGC 2903 is almost as bright in $\mathrm{H} \alpha$ as the whole bar, implying that it hosts similar SFRs (Alonso-Herrero et al. 2001; Leon et al. 2008).

Alternative SFR calibrations make use of the UV emission. We derived the integrated SFR, within the same region as for the calculation of the $\mathrm{SFR}_{\mathrm{H}_{\alpha}}$, using the UV emission as SF indicator. The SFR in the UV has been computed following the recipes given in Salim et al. (2007)

$$
S F R_{\mathrm{UV}}\left(M_{\odot} \mathrm{year}^{-1}\right)=1.08 \times 10^{-28} L_{\mathrm{FUV}}
$$

with $L_{\mathrm{FUV}}$ in $\mathrm{erg} \mathrm{s}^{-1} \mathrm{~Hz}^{-1}$. The star-formation rates calculated in this way infer a $S F R_{\mathrm{FUV}}=0.44 \pm 0.06 M_{\odot} \mathrm{yr}^{-1}$ for the bar region. The SFR calculated from both bands (cf. $S F R_{\mathrm{UV}}=0.44 \pm$ $0.06 M_{\odot} \mathrm{yr}^{-1}$ and $\left.S F R_{\mathrm{H} \alpha}=0.9 \pm 0.2 M_{\odot} \mathrm{yr}^{-1}\right)$ and the fluxes from which these SFR were obtained, are compatible with the results given by other works based on a large number of galaxies (i.e.; Lee et al. 2009; Salim et al. 2007). The SFR $\mathrm{UV}_{\mathrm{UV}}$ is a tracer of recent star-formation, averaged over some hundreds of Myrs (Calzetti et al. 2005; Kong et al. 2004), while the $\mathrm{SFR}_{\mathrm{H}_{\alpha}}$ probes the current star-formation. The SFRs derived in this paper are compatible with a number of star-formation histories, as derived from the Starburst 99 model. It is, however, interesting to date the age of the UV complexes with no $\mathrm{H} \alpha$ counterpart to shed some light into the origin of these structures. This is investigated in the next section.

\subsection{UV colours and cluster ages}

There is now a substantial amount of observational evidence that the FUV-NUV colours are very sensitive to the age of young 


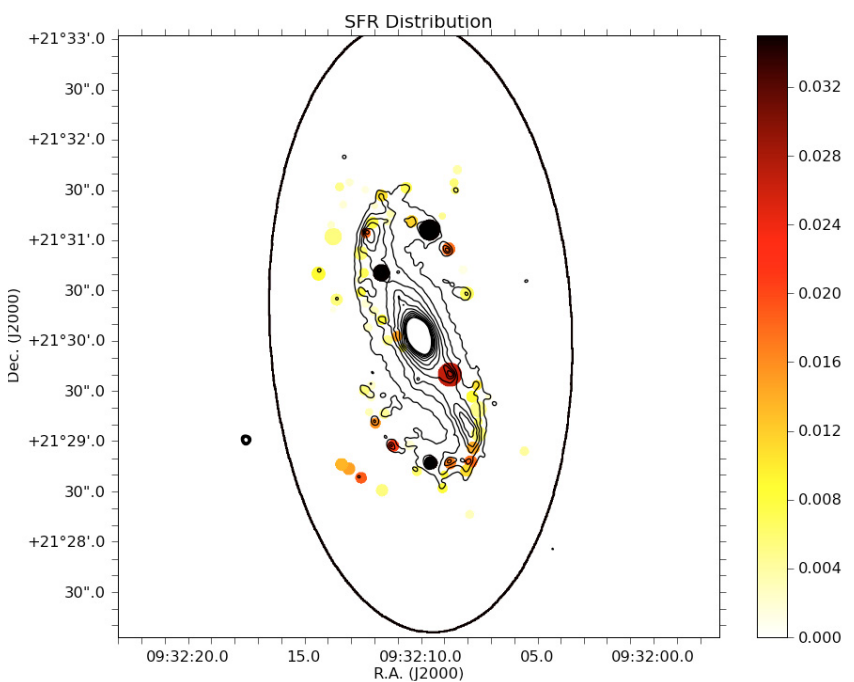

Fig. 7. SFR distribution in the bar region of NGC 2903. The size of the circles represents the $\mathrm{H}$ II region projected area. The SFR is given in $M_{\odot} \mathrm{yr}^{-1}$. The SFR was estimated from the measured $\mathrm{H} \alpha$ luminosity of the HII regions (see Sect. 5.3). The intensity contours trace the $3.6 \mu \mathrm{m}$ emission. The solid black line indicates the integration area for the calculation of the total SFR in the bar region (see Sect. 5.3)

stellar populations because of the rapid evolution of the most massive stars (i.e.; Bianchi et al. 2006; Calzetti et al. 2005). To estimate the ages of our catalogued UV regions, we used the FUV-NUV colours, which had been calculated using fluxes corrected for dust attenuation (Sect. 4.3). Figure 10 shows the range of UV colours in the bar of NGC 2903. The colours have a mean value of $0.3 \mathrm{mag}$ with a standard deviation of $0.27 \mathrm{mag}$. This range of values is typical of spiral galaxies (Thilker et al. 2005; Koribalski \& López-Sánchez 2009). Figure 9 shows the spatial distribution of the derived FUV-NUV regions. One of the most striking properties of the colour distribution are the redder regions located to the northwest and southeast of the galaxy nucleus and at the beginning of the spiral arm. These regions correspond mostly to those with the lowest attenuation. The regions located along the bar consist mostly of blue UV emitting regions.

As well as being age-dependent, the FUV-NUV colours are also strong tracers of metallicity; however, we can assume an approximately constant metallicity in the bar region of NGC 2903 (Pilyugin et al. 2004). With this assumption, the FUV-NUV colour is, as we already mentioned, a good tracer of age. To compare our results with the theoretical models and then derive the ages of the clusters, we model the evolution of a singlestar-formation burst using the Starburst99 population synthesis model (Leitherer et al. 1999). As input values, we used the Padova AGB stellar tracks with a metallicity of $Z=0.02$ (solar). For the IMF, we assumed a multi power-law parametrization with $\alpha=1.3$ for $0.1<M<0.5 M_{\odot}$, and $\alpha=2.35$ for higher masses up to $100 M_{\odot}$. We then derived the FUV-NUV colours from the simulated spectrum in multiple time-steps of between 1 Myr and 1 Gyr (see Fig. 11).

To estimate the UV region ages, we interpolated the modelled FUV-NUV colour versus age dependence to the observed UV colours. The colours of the bluer regions range from -0.18 to 0.15 , corresponding to an age of 3 to $10 \mathrm{Myr}$. The redder regions have colours ranging from 0.4 to 0.85 , which imply ages ranging from $\sim 150$ to $320 \mathrm{Myr}$. The latter group of colours corresponds to regions with significant amounts of neither $\mathrm{H} \alpha$ nor $24 \mu \mathrm{m}$ emission. The FUV-NUV colour

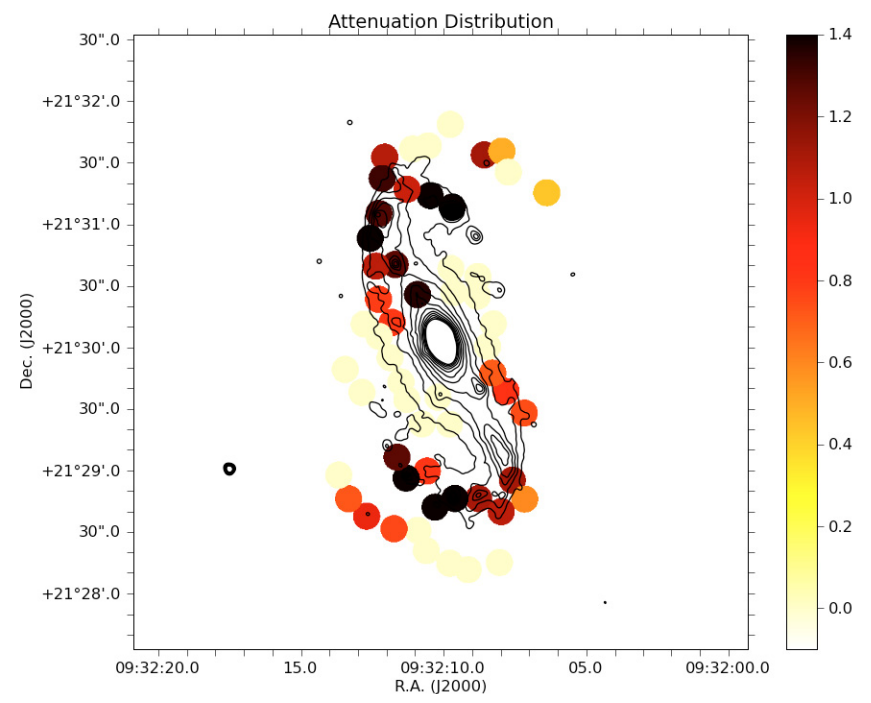

Fig. 8. Distribution of the $\mathrm{H} \alpha$ attenuation in NGC 2903. The circle size represents the integration apertures used for the UV catalogue. The intensity contours trace the $3.6 \mu \mathrm{m}$ emission.

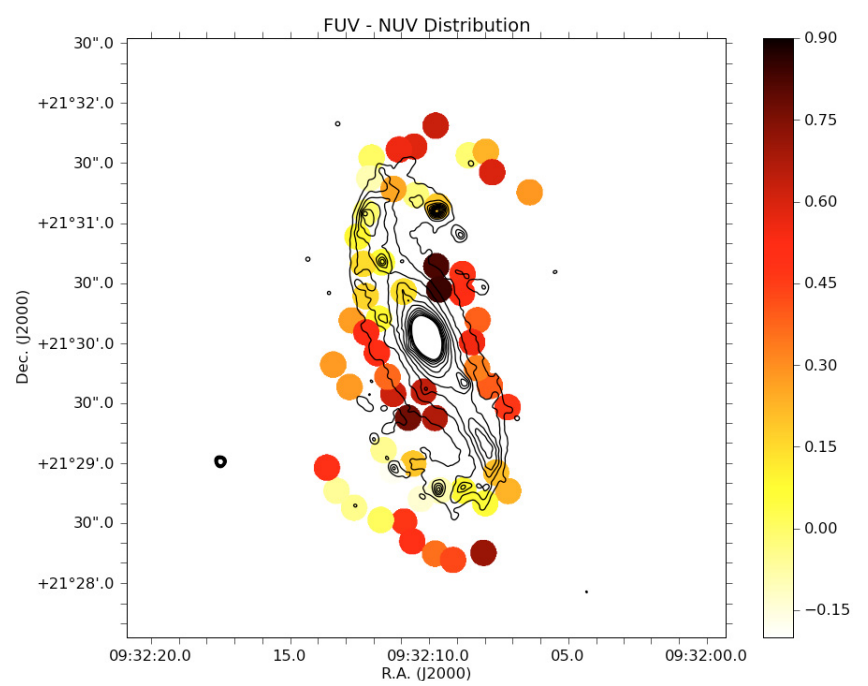

Fig. 9. Distribution of the UV colours in the NGC 2903 bar and its surrounding regions. The intensity contours trace the $3.6 \mu \mathrm{m}$ emission. A concentration of redder regions is clearly visible to the northeast and southwest of the nucleus. These regions correspond to the UV regions with no significant $\mathrm{H} \alpha$ counterpart.

remains constant around 0.2 implying ages between 10 and a little younger than 100 Myr.

\section{Discussion}

NGC 2903 is a clear example of a galaxy with very different morphologies as shown by different broad-band images. Although its bar is classified in the NIR with a bar strength of 3 (Laurikainen \& Salo 2002), corresponding to a relatively strong bar, and it is considered to be a classical bar, its morphology at shorter wavelengths being more similar to that of a patchy spiral disk. This clearly shows that to understand the SF history in bars it is necessary to obtain a panchromatic view.

To investigate the properties of SF in the NGC 2903 bar, we have at our disposal information about the distribution of gas in two different phases, namely the ionised gas and the colder $\mathrm{CO}(J=1-0)$. As for the stellar component, we are able to trace 


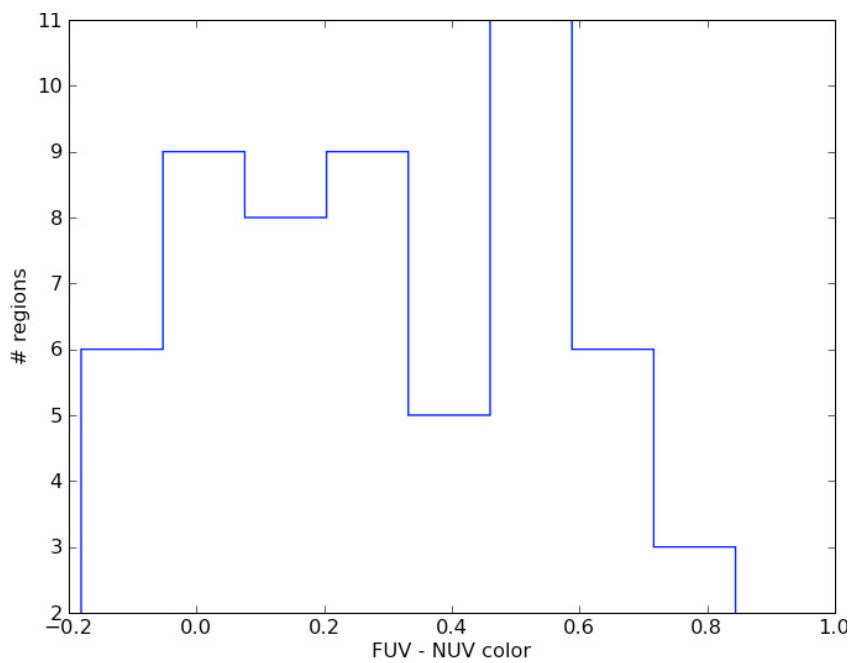

Fig. 10. FUV-NUV colour distribution for the catalogued UV regions in the bar zone of NGC 2903.

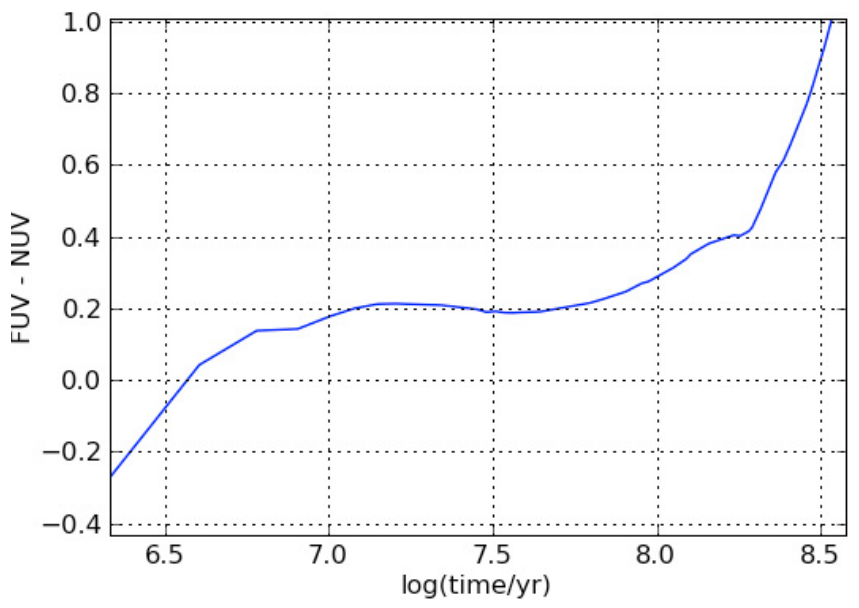

Fig. 11. Time evolution of the UV colour for a single starburst region. The model assumes Padova stellar tracks with $Z=0.02$ (solar) and an IMF constructed by a multi power-law parametrization with $\alpha=1.3$ for $0.1<M<0.5 M_{\odot}$ and $\alpha=2.35$ for higher masses up to $100 M_{\odot}$.

young stars up to $1 \mathrm{Gyr}$ with the UV information, the location of massive stars can be traced from the location of their H II regions, whereas intermediate and older stellar populations can be probed according to their distribution in both the $3.6 \mu \mathrm{m}$ and optical bands.

We observe that the CO $(J=1-0)$ distribution closely follows the emission in the $3.6 \mu \mathrm{m}$ band. There is a misalignment between the major axis of the bar derived from ellipse fitting of optical images (Sheth et al. 2002) and the $3.6 \mu \mathrm{m}$ major-axis light distribution. Both the $\mathrm{CO}(J=1-0)$ and the $3.6 \mu \mathrm{m}$ should therefore be on the leading side of the optical photometric axis of the bar. The $\mathrm{H} \alpha$ emission leads not only both the $\mathrm{CO}(J=1-0)$ and the $3.6 \mu \mathrm{m}$ emission but has a slightly different distribution, following a spiral pattern not so clearly seen in the CO $(J=1-0)$ and $3.6 \mu \mathrm{m}$ emission. The $\mathrm{H} \alpha$ emission also leads the dust lanes as traced by optical colour maps. Numerical models predict that the gaseous component (of both ionised and neutral gas) leads the stellar bar (Martin \& Friedli 1997).

This study started as a follow-up of the analysis carried out on the bar of NGC 1530 (Zurita \& Pérez 2008) to understand the physical processes determining star-formation in bars, where the importance of bar dynamics was clearly demonstrated. Both
$\mathrm{CO}(J=1-0)$ and dust are concentrated in regions where shocks and gas accumulation are induced by the bar potential. The distribution of the dust in the bar of NGC 1530 is much narrower than the distribution in NGC 2903. In NGC 1530, the dust lanes are straight and clearly delineated as opposed to the broken feathery morphology of the dust lanes in NGC 2903 (Fig. 2e). This may indicate that no strong shocks are present in the bar of NGC 2903, in contrast to the large velocity gradients perpendicular to the bar in NGC 1530 (Zurita et al. 2004), suggesting that shocks are driving the observed morphology in NGC 1530.

Streaming motions along the bar of NGC 2903 were detected by Leon et al. (2008) using $\mathrm{HCN}(1-0)$ as the kinematic tracer. An ongoing analysis of the velocity gradients in the ionised gas of NGC 2903, using the data published by Hernandez et al. (2005), will clarify whether strong velocity gradients are present or not in the bar of NGC 2903. Therefore, although the NIR emission suggest that NGC 2903 hosts a strong bar, the broken and wide structure of the dust lanes may well be evidence that it does not.

The presence of H II regions in both the trailing and leading sides of the dust lanes in NGC 1530 and an age gradient between both sets of H II regions, provides support to the hypothesis (Sheth et al. 2002) that massive stars form in the trailing side of the bar dust lane and move to the leading side. There are no H II regions on the trailing side of the bar of NGC 2903, and no trend in $\mathrm{H}$ II region age is found across the bar. This may indicate that the explanation of where star-formation is favoured, is not as straightforward as in NGC 1530, or at least is not as clearly driven by the strong bar dynamics.

The complexity of the SF properties in the bar of NGC 2903 is also illustrated by the presence of compact UV sources without significant $24 \mu \mathrm{m}$ and $\mathrm{H} \alpha$ emission. These regions are located symmetrically with respect to the centre of the galaxy and nearly perpendicular to the bar as well as in a spiral pattern. All of these regions have FUV-NUV colours compatible with ages of a couple of hundred Myr. The lack of $\mathrm{H} \alpha$ emission suggests that these locations are currently not encouraged by the bar potential to form stars. These stars might have formed somewhere else in the bar and migrated afterwards to the current position. But then a few questions would arise: (1) where did these stars form?; (2) are the stars that are currently forming likely to remain at similar locations?; or, (3) in contrast, were they formed during a more general SF burst possibly linked to the bar formation, or a merger? An ongoing analysis of both the $\mathrm{HI}$ and $\mathrm{H} \alpha$ velocity fields will shed some light onto the origin of the UV complexes.

The current SFR in the bar, as calculated from $\mathrm{H} \alpha$, is similar to those of other disk galaxies, although the bar contains a considerably high amount of dense cold gas, as traced by the HCN(1-0) (Leon et al. 2008). The recent SFR, as calculated from UV emission, is compatible with that of the current SFR.

We draw attention to the offsets of a few arcseconds between the emission peaks in the $24 \mu \mathrm{m}$ and the GALEX images. The $24 \mu \mathrm{m}$ emission is a good tracer of current star-formation, while UV emission is a "time-averaged" recent star-formation indicator (e.g.; Calzetti et al. 2005). The offsets found here (also seen in M 51 by Calzetti et al. 2005) indicate an evolutionary link between the sites where current star-formation is occurring and the position of the UV counterpart. Estimations of the rotation curve and the pattern speed would be necessary to determine whether the dynamical timescales are compatible with the ageing of the regions. 


\section{Summary and conclusions}

We have performed a detailed multiwavelength study of observations from UV to sub-millimeter wavelengths of the NGC 2903 bar and its surrounding regions. We have mapped and catalogued the $\mathrm{H}$ II regions of the bar and measured their $\mathrm{H} \alpha$ equivalent widths. Furthermore, we have obtained a catalogue of the UV emitting regions, and measured their peak locations with respect to those in the $\mathrm{H} \alpha$ image. The extinction has been estimated using the $\mathrm{H} \alpha$ and $24 \mu \mathrm{m}$ emission. Values of SFRs using both $\mathrm{H} \alpha$ and UV indicators have been calculated. We have estimated the age of the regions, using the $E W_{\mathrm{H} \alpha}$ and the FUV-NUV colour together with stellar population synthesis models. Our main results are:

- NGC 2903 is a morphologically complex galaxy. Both the near-infrared and the $\mathrm{CO}(J=1-0)$ band images detect a clear barred structure, whereas the $\mathrm{H} \alpha$ and UV maps show a patchy spiral-like structure.

- There are clear spiral-like UV complexes with no significant $\mathrm{H} \alpha, 24 \mu \mathrm{m}$, and $\mathrm{CO}(J=1-0)$ counterpart emission. These complexes are located northwest and Southeast of the bar within the inner 1 arcminute radius (corresponding to $\sim 2.5 \mathrm{kpc}$ ).

- The $\mathrm{H} \alpha$ emission along the bar, leads the CO. The $3.6 \mu \mathrm{m}$ and $\mathrm{CO}(J=1-0)$ emission trace each other, both leading the major axis of the optical light distribution.

- The $\mathrm{H} \alpha$ luminosities and $E W_{\mathrm{H} \alpha}$ of the bar $\mathrm{H}$ II regions are within typical ranges for $\mathrm{HII}$ regions in both bar and unbarred spirals. The spatial distribution of the $\mathrm{H} \alpha E W \mathrm{~s}$ does not correlate with any morphological feature in the bar.

- The average dust attenuation in the bar area of NGC 2903 is $A_{\mathrm{H} \alpha}=1.06$, and ranges from 0 to $1.5 \mathrm{mag}$.

- The FUV-NUV colour distribution is distributed into two regions. The bluer regions range from -0.18 to 0.15 implying an age between $\sim 3$ and $10 \mathrm{Myr}$. The redder regions have colours ranging from 0.4 to 0.85 , which imply ages ranging from 150 to $400 \mathrm{Myr}$. The latter correspond to regions with significant amounts of neither $\mathrm{H} \alpha$ nor $24 \mu \mathrm{m}$ emission.

- The SFRs of the bar region derived from both $\mathrm{H} \alpha$ and the UV emission are $0.9 \pm 0.2 M_{\odot} \mathrm{yr}^{-1}$ and $0.4 \pm 0.1 M_{\odot} \mathrm{yr}^{-1}$, respectively.

All these results suggest that some process triggered a SF burst a few hundred Myrs ago. Interestingly, we do detect some stellar clusters unrelated to the current SF locations, symmetrically located nearly perpendicular to the bar (in the inner $\sim 2.5 \mathrm{kpc}$ ). These regions might originate from this SF burst.

In a future paper, we will analyse the gas kinematics of the galaxy, with the aim of shedding some light on the origin (merger versus secular evolution) of these findings.

Acknowledgements. G. Popping acknowledges support by the Stichting Groninger Universiteitsfonds. I. Pérez acknowledges support by the Netherlands Organisation for Scientific Research (NWO, Veni-Grant 639.041.511). I. Pérez and A. Zurita acknowledge support from the Spanish Plan Nacional del Espacio de Ministerio de Educación y Ciencia (via grant C-CONSOLIDER AYA 2007-67625-C02-02). I. Pérez \& A. Zurita also thank the Junta de Andalucía for support through the FQM-108 project. We would like to thank Jorge Iglesias, Mónica Relaño, Ute Lisenfeld and Hervé Wozniak for the fruitful discussions.
We thank the referee for the very useful comments and suggestions that have greatly improved the manuscript.

\section{References}

Alonso-Herrero, A., Ryder, S. D., \& Knapen, J. H. 2001, MNRAS, 322, 757 Athanassoula, E. 1992, MNRAS, 259, 345

Bianchi, L., \& Efremova, B. V. 2006, AJ, 132, 378

Bianchi, L., Madore, B., Thilker, D., Gil de Paz, A., \& GALEX Science Team 2003, in BAAS, 35, 1354

Bianchi, L., Thilker, D. A., Burgarella, D., et al. 2005, ApJ, 619, L71

Bresolin, F., \& Kennicutt, Jr., R. C. 1997, AJ, 113, 975

Calzetti, D. 2001, PASP, 113, 1449

Calzetti, D., Armus, L., Bohlin, R. C., et al. 2000, ApJ, 533, 682

Calzetti, D., Kennicutt, Jr., R. C., Bianchi, L., et al. 2005, ApJ, 633, 871

Cedrés, B., Cepa, J., \& Tomita, A. 2005, ApJ, 634, 1043

Dale, D. A., Cohen, S. A., Johnson, L. C., et al. 2009, ApJ, 703, 517

Drozdovsky, I. O., \& Karachentsev, I. D. 2000, A\&AS, 142, 425

Engelbracht, C. W., Blaylock, M., Su, K. Y. L., et al. 2007, PASP, 119, 994

Fazio, G. G., Hora, J. L., Allen, L. E., et al. 2004, ApJS, 154, 10

Gonzalez Delgado, R. M., \& Perez, E. 1997, ApJS, 108, 199

Gordon, K. D., Rieke, G. H., Engelbracht, C. W., et al. 2005, PASP, 117, 503

Gordon, K. D., Engelbracht, C. W., Rieke, G. H., et al. 2008, ApJ, 682, 336

Helfer, T. T., Thornley, M. D., Regan, M. W., et al. 2003, ApJS, 145, 259

Hernandez, O., Carignan, C., Amram, P., Chemin, L., \& Daigle, O. 2005, MNRAS, 360, 1201

Hibbard, J. E., Bianchi, L., Thilker, D. A., et al. 2005, ApJ, 619, L87 Iglesias-Páramo, J., Buat, V., Takeuchi, T. T., et al. 2006, ApJS, 164, 38 Irwin, J. A., Hoffman, G. L., Spekkens, K., et al. 2009, ApJ, 692, 1447

Kennicutt, Jr., R. C. 1983, ApJ, 272, 54

Kennicutt, Jr., R. C. 1998, ARA\&A, 36, 189

Kennicutt, Jr., R. C., \& Kent, S. M. 1983, AJ, 88, 1094

Kennicutt, Jr., R. C., Edgar, B. K., \& Hodge, P. W. 1989, ApJ, 337, 761

Kennicutt, Jr., R. C., Armus, L., Bendo, G., et al. 2003, PASP, 115, 928

Kennicutt, Jr., R. C., Calzetti, D., Walter, F., et al. 2007, ApJ, 671, 333

Kennicutt, R. C., Hao, C., Calzetti, D., et al. 2009, ApJ, 703, 1672

Kong, X., Charlot, S., Brinchmann, J., \& Fall, S. M. 2004, MNRAS, 349, 769

Koribalski, B. S., \& López-Sánchez, Á. R. 2009, MNRAS, 400, 1749

Kormendy, J. 1979, ApJ, 227, 714

Kuchinski, L. E., Freedman, W. L., Madore, B. F., et al. 2000, ApJS, 131, 441

Laurikainen, E., \& Salo, H. 2002, MNRAS, 337, 1118

Lee, J. C., Kennicutt, R. C., Engelbracht, C. W., et al. 2008, in ed. J. G. Funes, \& E. M. Corsini, ASP Conf. Ser., 396, 151

Lee, J. C., Gil de Paz, A., Tremonti, C., et al. 2009, ApJ, 706, 599

Leitherer, C., Schaerer, D., Goldader, J. D., et al. 1999, ApJS, 123, 3

Leon, S., Jeyakumar, S., Pérez-Ramírez, D., et al. 2008, A\&A, 491, 703

Martin, P., \& Friedli, D. 1997, A\&A, 326, 449

Martin, D. C., Fanson, J., Schiminovich, D., et al. 2005, ApJ, 619, L1

Mayya, Y. D. 1994, AJ, 108, 1276

Pérez, I., Fux, R., \& Freeman, K. 2004, A\&A, 424, 799

Pérez-González, P. G., Kennicutt, Jr., R. C., Gordon, K. D., et al. 2006, ApJ, 648, 987

Pilyugin, L. S., Vílchez, J. M., \& Contini, T. 2004, A\&A, 425, 849

Regan, M. W., Thornley, M. D., Helfer, T. T., et al. 2001, ApJ, 561, 218

Relaño, M., \& Kennicutt, R. C. 2009, ApJ, 699, 1125

Relaño, M., Beckman, J. E., Zurita, A., Rozas, M., \& Giammanco, C. 2005, A\&A, 431, 235

Rieke, G. H., Young, E. T., Engelbracht, C. W., et al. 2004, ApJS, 154, 25

Rozas, M., Zurita, A., Heller, C. H., \& Beckman, J. E. 1999, A\&AS, 135, 145

Salim, S., Rich, R. M., Charlot, S., et al. 2007, ApJS, 173, 267

Schlegel, D. J., Finkbeiner, D. P., \& Davis, M. 1998, ApJ, 500, 525

Scoville, N. Z., Polletta, M., Ewald, S., et al. 2001, AJ, 122, 3017

Sheth, K., Vogel, S. N., Regan, M. W., et al. 2002, AJ, 124, 2581

Tamura, K., Jansen, R. A., \& Windhorst, R. A. 2009, AJ, 138, 1634

Thilker, D. A., Bianchi, L., Boissier, S., et al. 2005, ApJ, 619, L79

Thilker, D. A., Boissier, S., Bianchi, L., et al. 2007, ApJS, 173, 572

Verley, S., Corbelli, E., Giovanardi, C., \& Hunt, L. K. 2009, A\&A, 493, 453

von Hippel, T., \& Bothun, G. 1990, AJ, 100, 403

Werner, M. W., Roellig, T. L., Low, F. J., et al. 2004, ApJS, 154, 1

Zurita, A., \& Pérez, I. 2008, A\&A, 485, 5

Zurita, A., Relaño, M., Beckman, J. E., \& Knapen, J. H. 2004, A\&A, 413, 73 